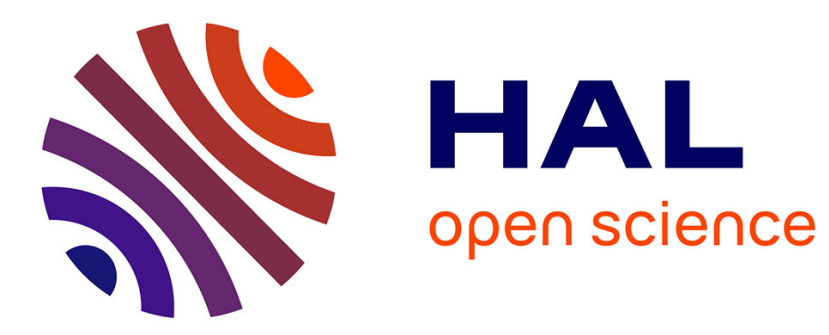

\title{
Parametric analysis for optimized piezoelectric bistable vibration energy harvesters
}

Thomas Huguet, Adrien Badel, Mickaël Null Lallart

\section{To cite this version:}

Thomas Huguet, Adrien Badel, Mickaël Null Lallart. Parametric analysis for optimized piezoelectric bistable vibration energy harvesters. Smart Materials and Structures, 2019, 28, 115009 (19pp). 10.1088/1361-665X/ab45c6 . hal-02305864

\section{HAL Id: hal-02305864 https://hal.science/hal-02305864}

Submitted on 4 Oct 2019

HAL is a multi-disciplinary open access archive for the deposit and dissemination of scientific research documents, whether they are published or not. The documents may come from teaching and research institutions in France or abroad, or from public or private research centers.
L'archive ouverte pluridisciplinaire HAL, est destinée au dépôt et à la diffusion de documents scientifiques de niveau recherche, publiés ou non, émanant des établissements d'enseignement et de recherche français ou étrangers, des laboratoires publics ou privés. 


\title{
Parametric analysis for optimized piezoelectric bistable vibration energy harvesters
}

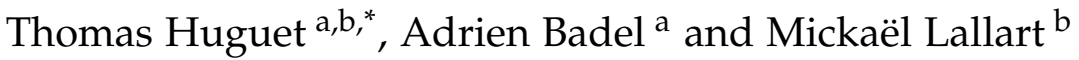 \\ a Univ. Savoie Mont-Blanc, SYMME, F-74000, Annecy, France \\ b Univ. Lyon, INSA-Lyon, LGEF, EA682, F-69621, Villeurbanne, France \\ * corresponding author: thomas.huguet@univ-smb.fr \\ adrien.badel@univ-smb.fr / mickael.lallart@insa-lyon.fr
}

\begin{abstract}
This study focuses on vibration energy harvesting with piezoelectric bistable inertial generators in order to provide an alternative or a support to chemical batteries in the power supply of isolated wireless sensors (leading to a better autonomy of these devices). The objective of the present work is to provide guidelines to optimize this kind of generator through the investigation of the influence of different parameters (load resistance, mass, stiffness, buckling level) on its frequency response. These guidelines will be obtained exploiting a new analytical model for piezoelectric bistable harvesters which will be constructed based on a recent model introduced for electromagnetic bistable harvesters. This model includes the study of subharmonic behaviors which can be used to enhance the global bandwidth of the harvester as well as a stability robustness criterion which allows a better prediction of experimental observations. The new model will be validated by experimental data and finally exploited to provide guidelines for piezoelectric bistable harvester optimization.
\end{abstract}

\section{Introduction}

This study is part of the current research concerning vibration energy harvesting with the aim of designing generators able to power wireless electronic devices thanks to the mechanical energy present in their environment [1]. These wireless devices usually work with chemical batteries which present a major limitation: the amount of energy embedded with the wireless device is limited which leads to several operations during its lifetime to change the empty batteries. This point is moreover intensified with the phenomenon of self-discharge of primary batteries especially in high temperature environment for instance. The use of vibration energy harvesters as an alternative to conventional batteries (or as a support to overcome their self-discharge) would therefore allow to increase the autonomy of wireless electronic devices converting continuously the ambient vibration to power them during their entire lifetime.

Several kinds of harvesters have been studied in the last twenty years [2]. Among them, inertial harvesters have the convenience of being fixable on a single point of the vibration source. Linear inertial harvesters (springmass) show interesting performance which nevertheless drastically collapse if the vibration frequency slightly moves from its resonance frequency. In order to increase the bandwidth proposed by vibration energy harvesters, part of the scientific community focused on nonlinear inertial harvesters [3] and more particularly on a promising architecture: the bistable harvester [4]. The study of bistable designs is still a topical issue in the community of energy harvesting $[5,6]$.

In order to optimize the performances of bistable harvesters, it is important to understand the influence of its different parameters on its frequency response as it was already proposed for linear harvesters [7]. The goal of this study is to develop and exploit a new analytical model for piezoelectric bistable harvesters in order to propose guidelines for their optimization. This model includes two new features: the study of subharmonic behaviors for which the mass oscillates periodically at a frequency $n$ times lower than the excitation and the stability robustness criterion which characterizes the capability of the different high orbits (inter-well motions) to handle big disturbances without falling on a low orbit (disturbances that can be found in real conditions). Those features appear to be relevant for optimization issues as the first one (subharmonic behaviors) can be used to enhance the global bandwidth of bistable harvesters [8] and the second one (stability robustness) allows better predictions of experimental measurements and observations [9]. The contribution of these two elements are illustrated in Figure 1 for an electromagnetic bistable harvester.

A few studies have already focused on optimization analysis for bistable harvesters but did not take into account neither the subharmonic behaviors nor the stability 


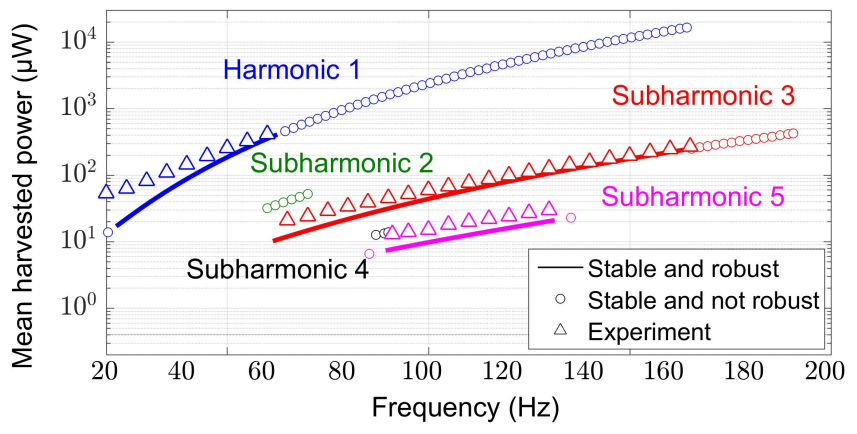

Figure 1: Contribution of subharmonic behaviors and stability robustness criterion on the frequency response of electromagnetic bistable harvester [9].

robustness criterion. Therefore, such results, although interesting, can be improved with the new model. Among these studies, Sneller et al. [10] highlighted the influence of the mass on the behavior of bistable harvesters: increasing the applied mass was found to broaden the response of the system and to lower the threshold for the onset of snap-through behaviour. Then Stanton et al. [11] focused on the mechanical and electrical damping: "there also exists an optimal impedance load and coupling for extracting maximum power for both interwell and intrawell oscillations". Finally, Panyam et al. [12] studied the influence of the load (time constant ratio), of the electromechanical coupling factor and of the buckling level (potential function): "while the time constant ratio has very little influence on the effective bandwidth of the harvester, increasing the electromechanical coupling and/or designing the potential function with deeper potential wells serve to shrink the effective bandwidth for a given level of excitation".

The present study is decomposed as follows. The first section proposes to establish the new analytical model for piezoelectric bistable harvesters based on a model recently proposed for electromagnetic bistable harvesters [9]. Then the second section introduces experimental data to validate this model. The third section exploits it to evaluate the influence of different parameters (load resistance, mass, stiffness, buckling level) on the frequency response of bistable harvesters. The conclusion section will finally summarize this study in the form of guidelines usable to optimize future piezoelectric bistable harvesters.

\section{Model construction}

The purpose of this section is to construct the analytical model for piezoelectric bistable harvester able to predict all its possible steady-state behaviors as a function of the excitation frequency (i.e., its frequency response). This model will be exploited later in this study to investigate the influence of the different parameters of the bistable harvester (mass, stiffness, buckling level, etc.) on its frequency response.

The bistable harvesters considered in this study is ob- tained with a buckled beam and a piezoelectric converter. The architecture is shown in Figure 2. It corresponds to a simplified schematic of the experimental prototype. This architecture includes a bistable oscillator with one degree of freedom and only one piezoelectric converter (in order to facilitate the assembly process). Contrary to the buckled beam bistable harvester introduced by Liu et al. [13], the piezoelectric converter is here located on the side of the oscillator rather than along the moving beams. This choice has been preferred to reduce its displacement that can damage the electrical connections while its deformations remain the same. The beams on either side of the mass are doubled to remove one unwanted degree of freedom: the rotation of the mass around the axis perpendicular to the plane of the diagram. The global stiffness $k$ shown in Figure 2 represents all the components of the bistable harvester that can store some elastic energy. Their respective stiffness are expressed in the same point to give the global stiffness $k$. The use of this global equivalent stiffness will lead to the generic Duffing type equations of piezoelectric bistable harvesters introduced later in Equation (5). Hence, the analysis which follows is valid for all the piezoelectric bistable harvesters governed by a Duffing type equation (a majority in the literature [4]) and does not depend on the particular architecture shown in Figure 2 which is given here as an example. It is noteworthy that the global stiffness $k$ can be easily identified experimentally knowing that bistable harvesters behave like linear harvesters for small perturbations. The global stiffness can then be determined through the identification of the resonance frequency of the bistable device for small perturbations as proposed in the experimental section. The mass $M$ represents the proof mass of the bistable harvester. The dynamic mass of the moving beams is neglected.

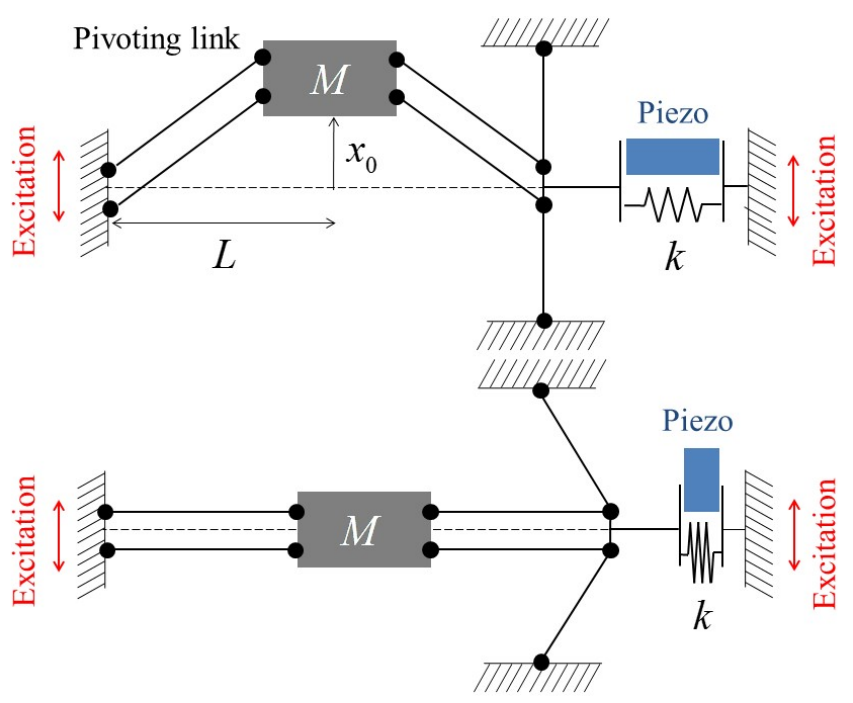

Figure 2: Architecture of the bistable harvester with piezoelectric coupling considered in this study. The different beams are supposed to be infinitely rigid. They are doubled on each side of the mass to prevent it from rotating (harvester with only one degree of freedom). The bistable harvester is illustrated in two different equilibrium positions: one of its two stable positions $x= \pm x_{0}$ and its unstable position $x=0$. 
The analytical model will focus on periodic steadystate behaviors of bistable harvester that are interesting for energy harvesting for a sinusoidal excitation: the common harmonic 1 high orbit for which the mass oscillates from one stable position to the other at the same frequency as the excitation and the subharmonic high orbits for which the mass oscillates from one stable position to the other at a frequency $n$ times lower than the excitation for subharmonic $n$. The harmonic 1 high orbit indeed presents the working point with the maximum harvested power and the subharmonic high orbits allow to increase the global bandwidth of the bistable harvester as they exist on frequency ranges on which the harmonic 1 high orbit is not reachable $[8,9]$.

The mathematical model will also characterize these steady-state behaviors with two criteria: their stability to small disturbances and their stability robustness. The first criterion, common in the study of dynamic systems, will split the behaviors in two categories by analyzing their response when a small disturbance is added: stable if maintained, unstable if not. The second criterion is calculated for stable high orbits and defines their ability to handle bigger disturbances without falling on a low orbit (disturbances that can be found in real conditions) [9]. The more robust the high orbit, the more capable to handle external disturbances without falling on a low orbit and therefore the easier to maintain over time in real conditions. A threshold will be experimentally defined for this criterion above which the high orbits will be considered as robust enough to be used for energy harvesting in real conditions.

The following construction is based on a model that have been recently introduced for electromagnetic bistable harvester [9]. It is here adapted to piezoelectric bistable harvester adding a new degree of freedom brought by the capacitance of the piezoelectric converter. The construction is here shorten only focusing on the differences with the previous model. For more details the reader may refer to the construction of this previous model [9].

\section{II.1. Formulation}

The mechanical equation of the bistable harvester (shown in Figure 2) is obtained analyzing the different forces applied on the mass: the absolute inertial force, the damping force (linear dependence on $\mathrm{x}$ ), the stiffness force and the force generated by the electromechanical conversion. The last two forces are projected on the vertical direction multiplying by $x / l$ and multiplied by a factor two to take into account the two forces generated by the beams on both sides of the mass.

$$
\begin{aligned}
& M(\ddot{x}+A \cos (\omega t))= \\
& -\mu \dot{x}-2 k \underbrace{\left(2 L-2 \sqrt{l^{2}-x^{2}}\right)}_{\text {piezo length variation }} \frac{x}{l}-2 \alpha v \frac{x}{l}
\end{aligned}
$$

where $x$ represents the position of the mass over time, $A$ and $\omega$ are the amplitude and the frequency of the excitation, $\mu$ the mechanical damping coefficient and $l$ the length of each of the four beams linked to the mass. The constant $\alpha$ represents the force factor of the piezoelectric converter and $v$ its voltage. The other variables are shown in Figure 2. In this study, the buckling coefficient $x_{0} / L$ is small compared to one. Considering this assumption and $l^{2}=x_{0}^{2}+L^{2}$, the mechanical equation becomes a Duffing-type equation:

$$
M(\ddot{x}+A \cos (\omega t)) \approx \frac{2 k}{L^{2}}\left(x_{0}^{2}-x^{2}\right) x-\mu \dot{x}-2 \alpha v \frac{x}{L}
$$

Part of the mechanical power of the bistable harvester is then converted in electrical power by the piezoelectric converter. In this study, this electrical power is entirely dissipated in a resistance $R$ directly connected to the piezoelectric element terminals. The power harvested by the device thus corresponds to the electrical power dissipated in this resistance. The electrical equation of the bistable harvester is obtained describing the electrical equilibrium between the piezoelectric converter (equivalent to a current generator proportional to the speed variation of the piezo length in parallel with a capacitance $C_{0}$ ) and the load resistance $R$ :

$$
C_{0} \dot{v}=\alpha \frac{\mathrm{d}}{\mathrm{d} t} \underbrace{\left(2 L-2 \sqrt{l^{2}-x^{2}}\right)}_{\text {piezo length variation }}-\frac{v}{R}
$$

Considering that the buckling coefficient $x_{0} / L$ is small compared to one and that $l^{2}=x_{0}^{2}+L^{2}$, the electrical equation becomes:

$$
C_{0} \dot{v} \approx 2 \frac{\alpha}{L} x \dot{x}-\frac{v}{R}
$$

The general equation set governing the behavior of the piezoelectric bistable harvester with low buckling level is therefore composed by Equation (2) and (4):

$$
\left\{\begin{array}{l}
\ddot{x}+\frac{\omega_{0}^{2}}{2}\left(\frac{x^{2}}{x_{0}^{2}}-1\right) x+\frac{\omega_{0}}{Q} \dot{x}+\frac{2 \alpha}{M L} x v=-A \cos (\omega t) \\
C_{0} \dot{v}=2 \frac{\alpha}{L} x \dot{x}-\frac{v}{R}
\end{array}\right.
$$

with

$$
\left\{\begin{array}{l}
\omega_{0}=\frac{x_{0}}{L} \sqrt{\frac{4 k}{M}} \\
Q=\frac{x_{0}}{L} \frac{\sqrt{4 k M}}{\mu}
\end{array}\right.
$$

The constant $\omega_{0}$ and $Q$ are the natural angular frequency and the mechanical quality factor respectively of the equivalent linear harvester which is obtained for small oscillations of the mass of the bistable harvester around one of its stable position $\left(x=x_{0}+\Delta x\right.$ with $\left.\Delta x<<x_{0}\right)$ [13]. The power harvested by the bistable harvester is 
equivalent to the power dissipated in the load resistance:

$$
\begin{aligned}
P_{\text {harvested }}(t) & =\frac{v(t)^{2}}{R} \\
P_{\text {mean harvested }} & =\frac{1}{T} \int_{0}^{T} \frac{v(t)^{2}}{R} d t
\end{aligned}
$$

In order to obtain the frequency response of the bistable harvester, it is now necessary to find all the steady-state behaviors which are solutions of Equation (5).

\section{II.2. Harmonic balance}

The focus is on behaviors useful for energy harvesting: the common harmonic 1 high orbit which presents the working point with the maximum harvested power and the subharmonic high orbits which allow to increase the global bandwidth of the bistable harvester as they exist on frequency ranges on which the harmonic 1 high orbit is not reachable $[8,9]$. As a reminder, subharmonic $n$ orbit is defined as a behavior for which the mass oscillates at frequency $n$ times lower than the excitation frequency. Examples of these behaviors are shown in Figure 3 (time signals), Figure 4 (phase portraits) and Figure 5 (Fourier series decomposition). These examples have been obtained with a numerical integration of Equation (5) for different initial conditions which are detailed in appendix. The values of the bistable harvester parameters correspond to the prototype introduced in the experimental section and are summarized in Table 2.

It is interesting to note on those figures that it exists a factor two between the fundamental frequency of the mass displacement and the preponderant frequency of the voltage. This phenomenon is caused by the symmetry of the bistable harvester with respect to the axes $x=0$ : the piezoelectric converter deforms in the same way for positive $x$ and for negative $x$. Thus, the voltage maxima (corresponding to the elongation maxima of the piezoelectric converter) are reached for all the displacement extrema of the mass (maxima and minima). The voltage minima (corresponding to the compression maxima of the piezoelectric converter) are reached each time the mass passes through $x=0$.

It is also worth noting on Figure 5 that the mass displacement of the subharmonic high orbits with an odd order (1, 3 and 5) is only composed of odd harmonics of the fundamental frequency $(\mathrm{h} 3, \mathrm{~h} 5, \mathrm{~h} 7 . .$.$) . For these$ behaviors, the voltage is composed of a fundamental frequency twice higher than the mass displacement and all its harmonics. The mass displacement of the subharmonic high orbits with an even order (2 and 4$)$ is composed of all the harmonics of the fundamental frequency and a significant continuous component. This time, the voltage of these behaviors is composed of the same harmonics as the mass displacement (with a preponderant frequency twice higher than the fundamental frequency of the mass displacement).

This difference between odd and even subharmonic orbits is directly linked to the bistability of the harvester and especially to the force applied by the spring to the mass which can be expressed as a polynomial equation involving only odd terms $A x^{3}+B x$ with $(A, B)$ constants (Equation (5)). If $f$ is the fundamental frequency of the mass position, the spring force only creates odd harmonics of $f$ when the continuous component of the mass position is zero and creates all the harmonics of $f$ otherwise. However, a behavior can only be maintained in a steady-state if the excitation can bring energy to the harvester by interacting with one of the harmonics of the mass position. For odd subharmonic behaviors, the excitation must interact with an odd harmonic of the mass
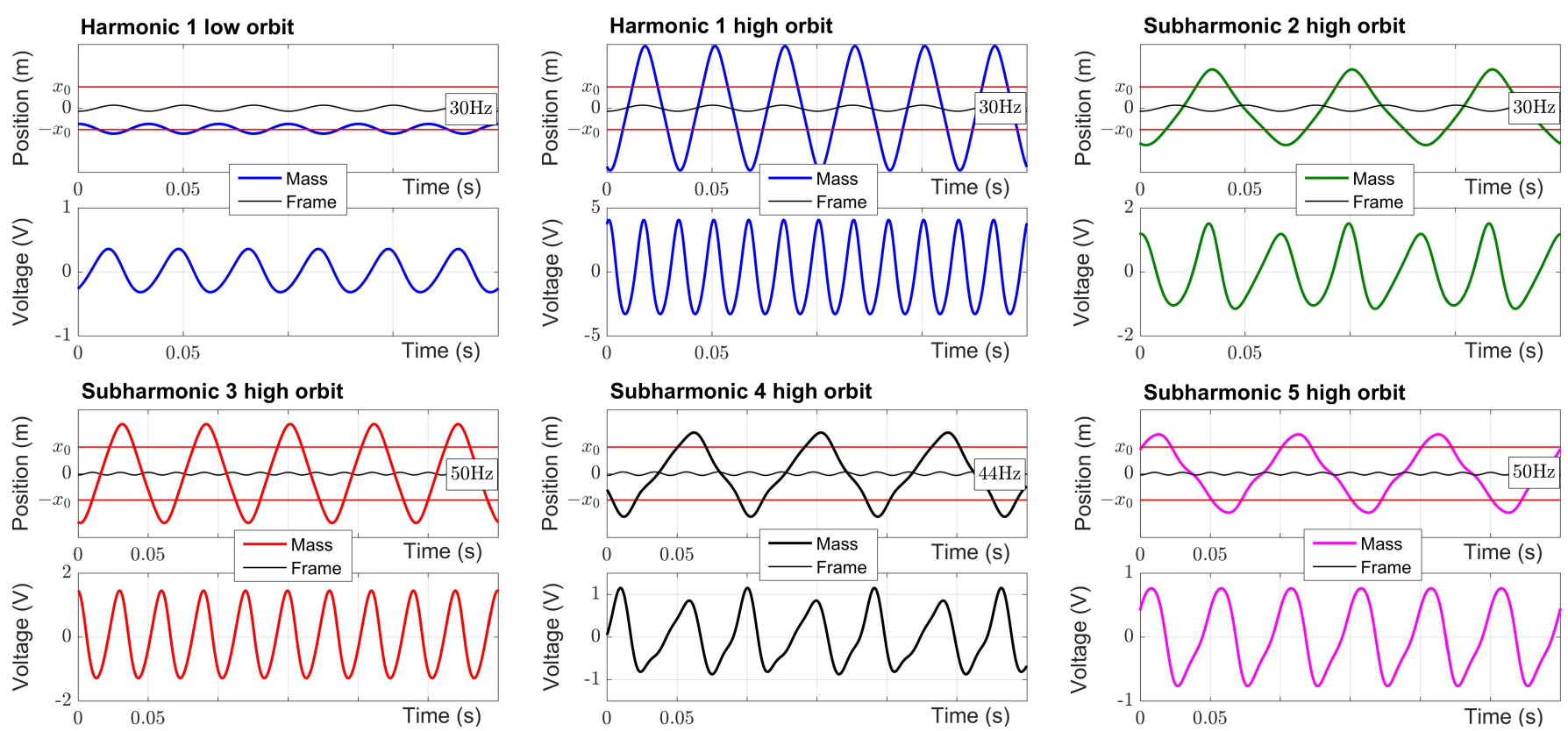

Figure 3: Examples of time signal of steady-state behaviors sought (numerical study). 

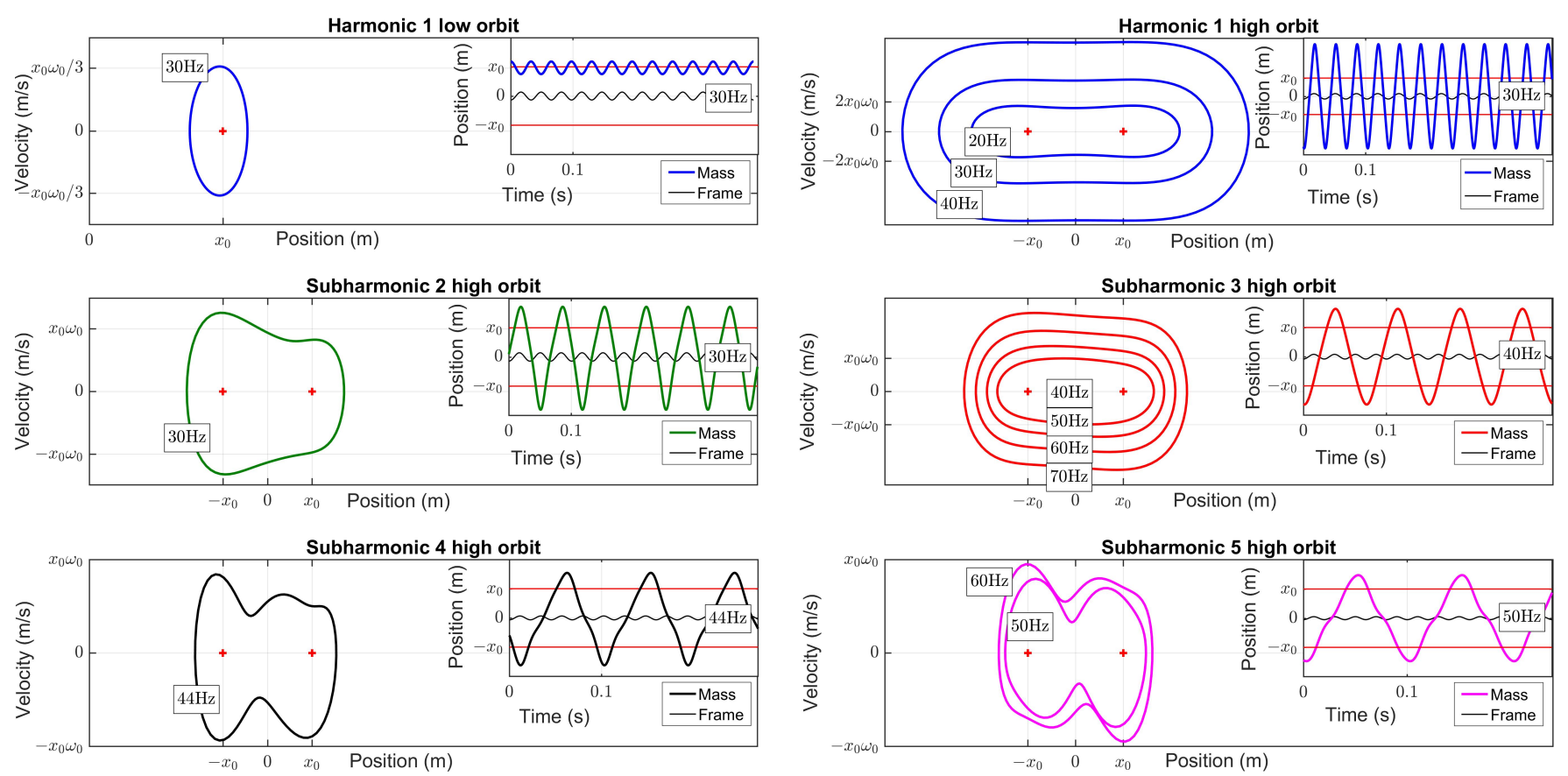

Figure 4: Example of displacement-velocity phase portrait of the steady-state behaviors sought (numerical study) with their respective Poincaré map (black dots).

position (see Figure 5). For even subharmonic behaviors, it must interact with an even harmonic of the mass position (see Figure 5). Thus, only the even subharmonic behaviors have a continuous component on the mass position which implies that their whole displacement oscillations are shifted up or down and are no longer symmetric with respect to the axes $x=0$ contrary to odd subharmonic behaviors. The piezoelectric voltage is linked to a term $A \dot{x} x$ with $A$ constant (Equation (5)). Therefore, its fundamental component is $2 f$ and it is only composed of even harmonics of $f$ for odd subharmonic behaviors and of all the harmonics of $f$ for even subharmonic behaviors.

The general form of the solution (corresponding to subharmonic $n$ behavior) is thus approached by two Fourier series truncated to order $N$ in order to be able to apply the classical method of harmonic balance [4] Each of them is therefore composed of a fundamental component with an angular frequency $\omega / n$ followed by all its harmonics $h \omega / n$ with $h \in \llbracket 1, N \rrbracket$ and constant
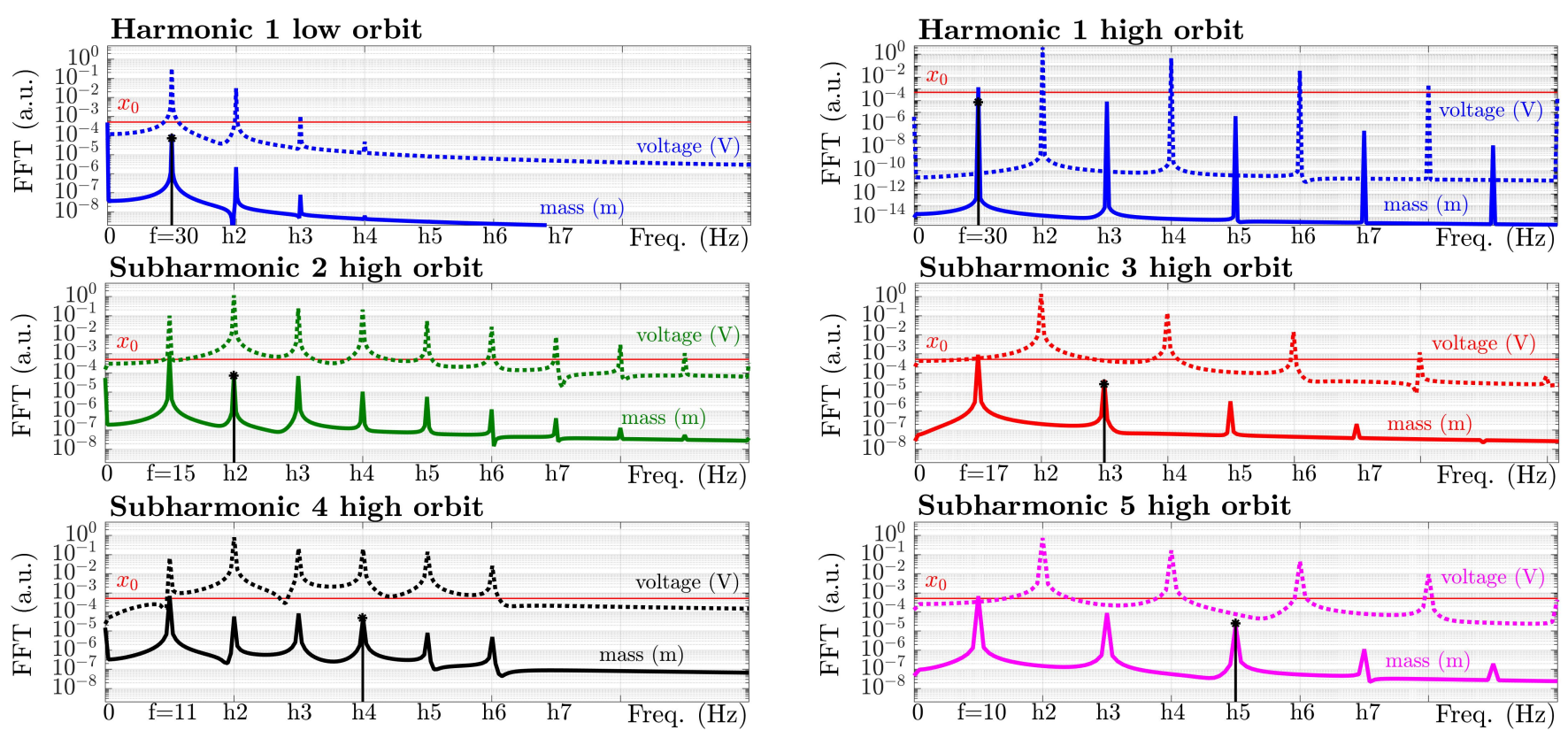

Figure 5: Example of Fourier series decomposition of the steady-state behaviors sought (numerical study). On the X-axis, " $f$ " refers to the fundamental component of the mass displacement and " $h$ " refers to its different harmonics. The monochromatic excitation is represented by a vertical black line (displacement of the frame). 
terms $a_{0}$ and $p_{0}$ :

$$
\left\{\begin{array}{l}
x(t)=a_{0}+\sum_{h=1}^{N}\left\{a_{h} \cos \left(h \frac{\omega}{n} t\right)+b_{h} \sin \left(h \frac{\omega}{n} t\right)\right\} \\
v(t)=p_{0}+\sum_{h=1}^{N}\left\{p_{h} \cos \left(h \frac{\omega}{n} t\right)+q_{h} \sin \left(h \frac{\omega}{n} t\right)\right\}
\end{array}\right.
$$

where the constant terms $a_{0}$ and $p_{0}$ corresponds respectively to the time averages of mass displacement and voltage at the resistance terminals. If $a_{0}$ is zero, the solution is a high orbit. If $a_{0}$ is close to $x_{0}$ or $-x_{0}$, the solution is a low orbit. Since the desired response is in steady-state, the $4 N+2$ unknown $\left(a_{0}, a_{h}, b_{h}, p_{0}, p_{h}, q_{h}\right), h \in \llbracket 1, N \rrbracket$ are independent of time.

Equation (9) is injected in Equation (5) governing the behavior of bistable harvester. An approximated solution of the system can then be found thanks to the harmonic balance method [9]. Each approximated solution $\left(x_{s}, v_{S}\right)$ found with the harmonic balance method is defined by a unique set $\left(a_{s 0}, a_{s h}, b_{s h}, p_{s 0}, p_{s h}, q_{s h}\right)$ with $h \in \llbracket 1, N \rrbracket$. These unique sets all correspond to possible steady-state subharmonic $n$ behaviors of the bistable harvester for an excitation $A \cos (\omega t)$.

The frequency response of the bistable harvester can therefore be obtained solving Equation (5) with this method for different values of $n$ (giving the different subharmonic $n$ behaviors including harmonic 1 for $n=1$ ) and for different angular frequencies of the excitation $\omega$.

This method has often been applied in the literature to study the harmonic 1 behavior with only one term in the truncated Fourier series $(N=1)$. In our case, $N$ has been chosen bigger than one to reduce the error on the solution sought and to reveal the subharmonic behaviors. It is determined with the following method: $N$ is increased until the difference between the solution found and the solution for $N-1$ becomes negligible. This method converged to $N=6$.

\section{II.3. Stability to small disturbances}

Stability to small disturbances is then evaluated for all steady-state behaviors $\left(x_{s}, v_{s}\right)$, corresponding to approximated solutions of Equation (5). A small disturbance $(z, w)$ is therefore added to the studied behavior as a pulse at the instant $t=t_{0}$ :

$$
\left\{\begin{array}{l}
x(t)=x_{s}(t)+z(t) \\
v(t)=v_{S}(t)+w(t)
\end{array}\right.
$$

with

$$
\left\{\begin{array}{l}
\|z(t)\|<<\left\|x_{s}(t)\right\| \\
\|w(t)\|<<\left\|v_{S}(t)\right\|
\end{array}\right.
$$

and

$$
\text { pulse }=\left(\begin{array}{c}
z\left(t_{0}\right) \\
\dot{z}\left(t_{0}\right) \\
w\left(t_{0}\right)
\end{array}\right)
$$

The solution $\left(x_{s}, v_{s}\right)$ is stable to small disturbances if and only if $(z, w)$ tend towards 0 . Equation (10) is injected in Equation (5) governing the behavior of the bistable harvester and linearized with respect to $(z, w)$ to give the following differential equation governing the behavior of the disturbance:

$$
\left(\begin{array}{c}
\dot{z}(t) \\
\ddot{z}(t) \\
\dot{w}(t)
\end{array}\right)=\underline{\underline{M}}(t)\left(\begin{array}{c}
z(t) \\
\dot{z}(t) \\
w(t)
\end{array}\right)
$$

with

$$
\begin{aligned}
& \underline{\underline{M}}(t)= \\
& {\left[\begin{array}{ccc}
0 & 1 & 0 \\
\frac{\omega_{0}^{2}}{2}-\frac{3 \omega_{0}^{2}}{2 x_{0}^{2}} x_{s}(t)^{2}-\frac{2 \alpha}{M L} v_{s}(t) & -\frac{\omega_{0}}{Q} & -\frac{2 \alpha}{M L} x_{s}(t) \\
\frac{2 \alpha}{C_{0} L} \dot{x}_{s}(t) & \frac{2 \alpha}{C_{0} L} x_{s}(t) & -\frac{1}{C_{0} R}
\end{array}\right]}
\end{aligned}
$$

The stability to small disturbances can now be evaluated with the Floquet theory and the Lyapunov exponents. The stability of the solutions $\left(x_{s}, v_{s}\right)$ is given by the eigenvalues $\left(\beta_{1}, \beta_{2}, \beta_{3}\right)$ of the resolvent matrix $\underline{\underline{R}}(2 T, 0)$, obtained after two numerical integrations of Equation (13) [9]:

$$
\underline{\underline{R}}(2 T, 0)=\left[\left(\begin{array}{c}
z_{1}(2 T) \\
\dot{z_{1}}(2 T) \\
w_{1}(2 T)
\end{array}\right)\left(\begin{array}{c}
z_{2}(2 T) \\
\dot{z_{2}}(2 T) \\
w_{2}(2 T)
\end{array}\right)\left(\begin{array}{c}
z_{3}(2 T) \\
\dot{z_{3}}(2 T) \\
w_{3}(2 T)
\end{array}\right)\right]
$$

with

$$
\begin{aligned}
& \left(\left(\begin{array}{c}
z_{1}(0) \\
\dot{z}_{1}(0) \\
w_{1}(0)
\end{array}\right)=\left(\begin{array}{l}
1 \\
0 \\
0
\end{array}\right) \underset{\text { Equation (13) from } 0 \text { to } 2 T}{\stackrel{\text { numerical integration of }}{\longrightarrow}}\left(\begin{array}{c}
z_{1}(2 T) \\
\dot{z}_{1}(2 T) \\
w_{1}(2 T)
\end{array}\right)\right. \\
& \left\{\left(\begin{array}{c}
z_{2}(0) \\
z_{2}(0) \\
w_{2}(0)
\end{array}\right)=\left(\begin{array}{l}
0 \\
1 \\
0
\end{array}\right) \underset{\text { Equation (13) from } 0 \text { to } 2 T}{\stackrel{\text { numerical intégration }}{\longrightarrow}}\left(\begin{array}{c}
z_{2}(2 T) \\
z_{2}(2 T) \\
w_{2}(2 T)
\end{array}\right)\right. \\
& \left(\begin{array}{c}
z_{3}(0) \\
\dot{z_{3}}(0) \\
w_{3}(0)
\end{array}\right)=\left(\begin{array}{l}
0 \\
0 \\
1
\end{array}\right) \underset{\text { Equation (13) from } 0 \text { to } 2 T}{\stackrel{\text { numerical intégration }}{\longrightarrow}}\left(\begin{array}{c}
z_{3}(2 T) \\
\dot{z_{3}}(2 T) \\
w_{3}(2 T)
\end{array}\right)
\end{aligned}
$$

and

$$
\left(x_{s}, v_{s}\right) \text { stable } \Leftrightarrow\left\{\begin{array}{l}
\left|\beta_{1}\right|<1 \\
\left|\beta_{2}\right|<1 \\
\left|\beta_{3}\right|<1
\end{array}\right.
$$

\section{II.4. Stability robustness}

The stability robustness will now be evaluated for all the high orbits defined as stable to small disturbances. This criterion will define the ability of these high orbits to handle bigger disturbances without falling on a low orbit (disturbances that can be found in real conditions). The more robust the high orbit, the more capable to handle external disturbances without falling on a low orbit and therefore the easier to maintain over time in real conditions. A threshold will be defined for this 
criterion above which the high orbits will be considered as robust enough to be used for energy harvesting in real conditions.

Hence, a disturbance $(z, w)$ is added to the stable (to small disturbances) high orbit $\left(x_{S}, v_{S}\right)$ as a speed pulse $\Lambda$ at the instant $t=t_{0}$. This pulse is not considered as negligible anymore:

$$
\left\{\begin{array}{l}
x(t)=x_{s}(t)+z(t) \\
v(t)=v_{s}(t)+w(t)
\end{array}\right.
$$

and

$$
\text { pulse }=\left(\begin{array}{c}
z\left(t_{0}\right) \\
\dot{z}\left(t_{0}\right) \\
w\left(t_{0}\right)
\end{array}\right)=\left(\begin{array}{c}
0 \\
\Lambda \\
0
\end{array}\right)
$$

The stability robustness is evaluated calculating the minimum value of the pulse $\Lambda$ which leads to a destabilization of the high orbit. Thus, the high orbit remains stable ( $z$ and $w$ tend towards 0 ) if $\Lambda<\Lambda_{\min }$ and the high orbit becomes unstable ( $z$ and $w$ tend towards infinity) if $\Lambda>\Lambda_{\text {min }}$. More precisely, the stability robustness is defined by the ratio between the minimum energy needed to destabilize the high orbit and the average amount of energy provided by the excitation during one period (normative value):

$$
\text { Robustness }=\frac{\frac{1}{2} M \Lambda_{\text {min }}^{2}}{E_{\text {excitation }}}
$$

with

$$
E_{\text {excitation }}=-\frac{1}{n} \int_{0}^{T} M A \cos (\omega t) \dot{x_{s}} d t
$$

The next step is to determine the expression of $\Lambda_{\min }$. Equation (18) is injected in Equation (5) governing the behavior of the bistable harvester to give the following differential equation governing the behavior of the disturbance:

$$
\begin{aligned}
\left(\begin{array}{c}
\dot{z}(t) \\
\ddot{z}(t) \\
\dot{w}(t)
\end{array}\right) & =\underbrace{\underline{\underline{M}(t)\left(\begin{array}{c}
z(t) \\
\dot{z}(t) \\
w(t)
\end{array}\right)}}_{\text {linear term }} \\
+ & \underbrace{\left(-\frac{3 \omega_{0}^{2}}{2 x_{0}^{2}} x_{s}(t) z(t)^{2}-\frac{\omega_{0}^{2}}{2 x_{0}^{2}} z(t)^{3}-\frac{2 \alpha}{M L} z(t) w(t)\right.}_{\frac{2 \alpha}{C_{0} L} z(t) \dot{z}(t)})
\end{aligned}
$$

The matrix $M(t)$ is identical to the one introduced in the small disturbance analyses in Equation (14). The minimal speed pulse $\Lambda_{\text {min }}$ which leads to the divergence of the disturbance $(z, w)$ can be calculated thanks to a reductio ad absurdum applied on this differential equation. The expression of the minimal pulse $\Lambda_{\text {min }}$ which leads to destabilize the high orbit ends up to be [9]:

$$
\Lambda_{\min }=\frac{\delta_{0}-B \delta_{0}^{2}-D \delta_{0}^{3}}{[\underline{\underline{C}}]_{(1,2)}+\frac{2 \alpha}{C_{0} L \lambda} \delta_{0}[\underline{\underline{C}}]_{(1,3)}}
$$

with

$$
\left\{\begin{array}{l}
\delta_{0}=\frac{-B+\sqrt{B^{2}+3 D}}{3 D} \\
B=\left(\frac{3 \omega_{0}^{2}}{2 x_{0}^{2}} \max _{t_{0} \in[0, T]}|\Psi|+\frac{2 \alpha}{M L \lambda}\right)[\underline{\underline{C}}]_{(1,2)}+\frac{2 \alpha}{C_{0} L \lambda}[\underline{\underline{C}}]_{(1,3)} \\
D=\frac{\omega_{0}^{2}}{4 \lambda x_{0}^{2}}[\underline{\underline{C}}]_{(1,2)} \\
{[\underline{\underline{C}}]_{(i, j)}=\max _{\substack{t_{0} \in[0, T] \\
t \in\left[t_{0}, t_{0}+2 T\right]}}\left|e^{\lambda\left(t-t_{0}\right)}\left[\underline{\underline{R}}\left(t, t_{0}\right)\right]_{(i, j)}\right|}
\end{array}\right.
$$

and

$$
\left\{\begin{aligned}
\lambda & =-\frac{\max \left(\left(\ln \left(\beta_{1}\right), \ln \left(\beta_{2}\right), \ln \left(\beta_{3}\right)\right)\right.}{2 T} \\
\Psi & =\sum_{k=1}^{N}\left\{\frac{a_{s k}}{\lambda^{2}+\omega_{k}^{2}}\left(\lambda \cos \left(\omega_{k} t_{0}\right)-\omega_{k} \sin \left(\omega_{k} t_{0}\right)\right)\right. \\
& \left.+\sum_{k=1}^{N} \frac{b_{s k}}{\lambda^{2}+\omega_{k}^{2}}\left(\lambda \sin \left(\omega_{k} t_{0}\right)+\omega_{k} \cos \left(\omega_{k} t_{0}\right)\right)\right\} \\
T & =2 \pi n / \omega \\
\omega_{k} & =k \omega / n
\end{aligned}\right.
$$

The stability robustness can now be calculated for all the high orbits defined as stable to small disturbances knowing the minimal pulse $\Lambda_{\text {min }}$ which leads to their fall on a low orbit.

The mathematical model able to predict the frequency response of piezoelectric bistable harvesters is now complete. The next section provides experimental data to validate it. All the results (theoretical and experimental) are presented at the end of the next section in Figure 9.

\section{Model validation with experimental data}

\section{III.1. Prototype and identification}

The experimental analysis conducted to validate the model built in the previous section has been done with the prototype shown in Figure 6. The actuator visible on the right of the top picture is not used in this study. Its stiffness is high compared to the stiffness of the piezoelectric converter. As long as this actuator is not powered, it thus does not affect the behavior of the bistable harvester. The prototype is buckled thanks to a nut which (when it is screwed on) causes a translation of the screw linked to the actuator. The beams on both sides of the mass are doubled in order to prevent the mass from rotating.

Technically speaking, the prototype was obtained by cutting a block of APX4 steel by electrical discharge machining to make the mass, the beams and the frame appear. The piezoelectric converter (and the actuator) is then assembled. The piezoelectric converter is manufactured by Cedrat Technologies under the reference APA 


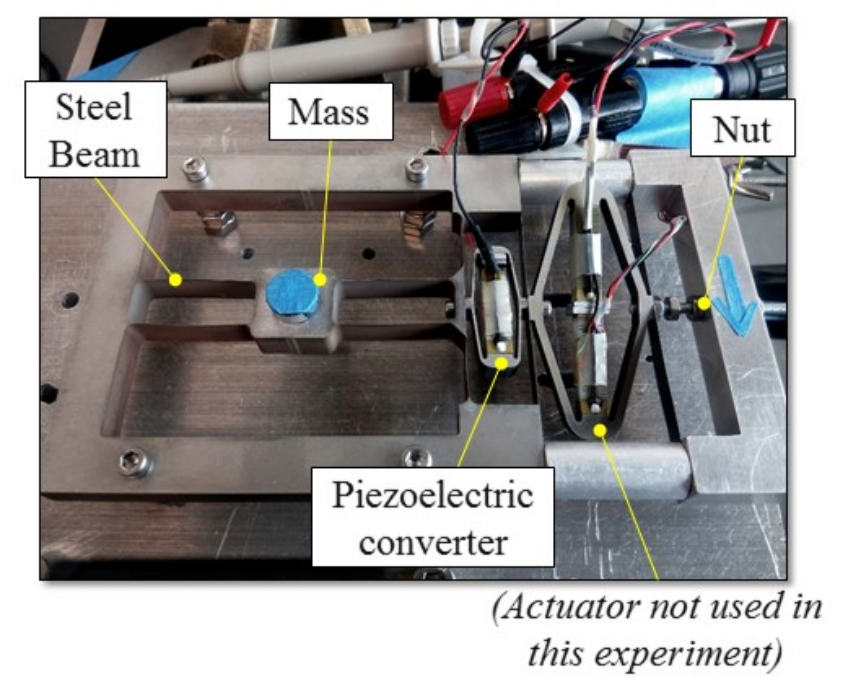

Figure 6: Piezoelectric bistable harvester made with four beams buckled thanks to the nut.

$120 S$ (Amplified Piezoelectric Actuator). It consists of two elements: an elliptical stainless steel shell and a stack of inter-digitated piezoelectric ceramics placed on the long axis of this shell. The elliptical shape of the shell acts as a mechanical transformer. It amplifies the stresses applied to its small axis along its long axis. On the other hand, it reduces the deformations applied to its small axis along its long axis. This APA $120 \mathrm{~S}$ thus optimizes the use of piezoelectric ceramics, which can support high forces but small deformations. The main dimensions of the prototype are detailed in Table 1.

This prototype corresponds to the simplified schematic introduced in Figure 2. The pivoting links are ensured by the flexibility of the thin steel beams (flexible connections). The global stiffness $k$ shown in Figure 2 thus includes, in this particular case, (i) the stiffness of the piezoelectric converter and (ii) the stiffness of the flexible beams. The latter is here negligible as the potential energy stored in the flexible bonds is small compared to the potential energy stored in the piezoelectric converter. The stiffness of the piezoelectric converter is itself composed by the stiffness of the piezoelectric stack, the stiffness of the elliptical shell and it also includes the effect of the mechanical transformation caused by the shell which amplifies the stresses applied to its small axis along its long axis and reduces the deformations applied to its small axis along its long axis. This transformation

Table 1: Prototype main dimensions.

\begin{tabular}{lcc}
\hline \hline Element & Value & Unit \\
\hline Inertial mass & 17.3 & $\mathrm{~g}$ \\
APX4 steel block thickness & 8.0 & $\mathrm{~mm}$ \\
Steel mass & $16 \times 16 \times 8.0$ & $\mathrm{~mm}^{3}$ \\
Beams thickness & 0.070 & $\mathrm{~mm}$ \\
Horizontal beams length & 25 & $\mathrm{~mm}$ \\
Vertical beams length & 17 & $\mathrm{~mm}$ \\
Piezoelectric stack & $20 \times 5.0 \times 9.0$ & $\mathrm{~mm}$ \\
Piezoelectric converter stiffness $k$ & 0.30 & N. $\mu \mathrm{m}^{-1}$ \\
\hline \hline
\end{tabular}

therefore affects the global stiffness of the piezoelectric converter.

These flexible bonds have a negligible effect on the stiffness and damping of the bistable harvester and can therefore be modeled as pure pivoting links. Indeed, (i) the potential energy stored in these flexible bonds is small compared to the potential energy stored in the Piezo harvester (for low buckling configuration) and (ii) the mechanical losses in these bonds are negligible thanks to the material used (APX4 steel).

The model parameters $\left(\omega_{0}, Q, C_{0}, \alpha\right)$ have been identified measuring the complex admittance $\underline{Y}$ of the piezoelectric converter (mounted on the prototype) around the angular frequency $\omega_{0}$ of the bistable harvester without any excitation [14]. The different parameters are then identified adjusting the experimental admittance to the theoretical one obtained for a small voltage excitation of the piezoelectric converter inducing small oscillations of the mass around one of its stable positions: $x=x_{0}+\Delta x$ with $\Delta x<<x_{0}$. This identification is shown in Figure 7 . The expression of the theoretical admittance being for the bistable harvester:

$$
\left\{\begin{array}{l}
\underline{Y}=\frac{\underline{I}}{\underline{V}}=j C_{0} \omega\left(1+\frac{k_{\mathrm{em}}^{2}}{1-\left(\omega / \omega_{0}\right)^{2}+j \frac{\omega / \omega_{0}}{Q}}\right) \\
k_{\mathrm{em}}^{2}=4\left(\frac{x_{0}}{L}\right)^{2} \frac{\alpha^{2}}{M C_{0} \omega_{0}^{2}}
\end{array}\right.
$$

The other model parameters $\left(M, L, x_{0}\right)$ are directly measured on the prototype. All the parameters and their identified values for the prototype are summarized in Table 2.
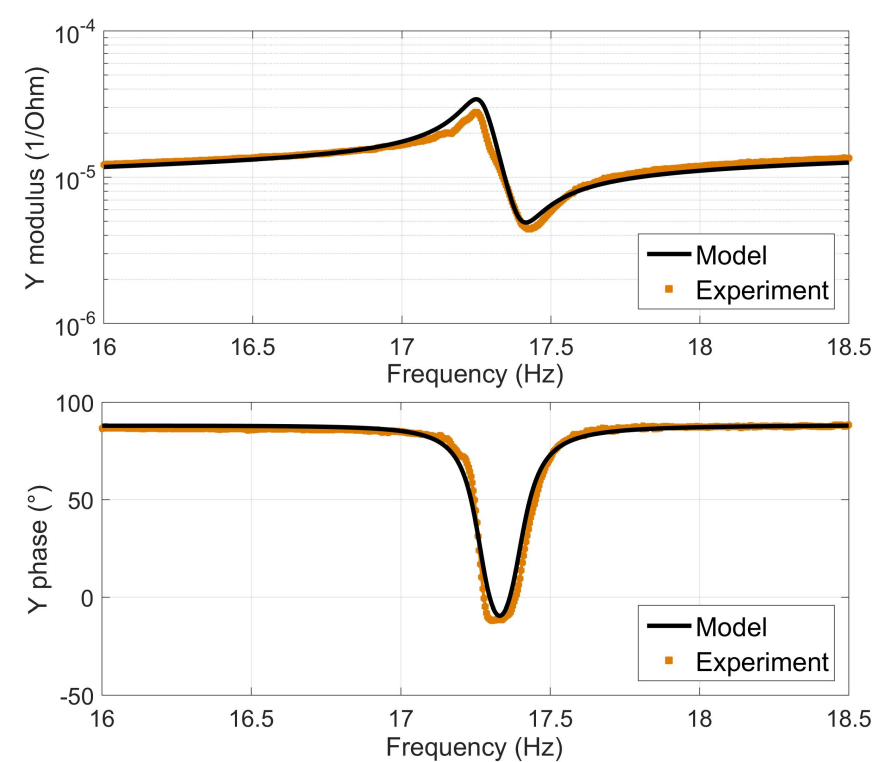

Figure 7: Admittance adjustment for the parameters $\left(\omega_{0}, Q, C_{0}, \alpha\right)$ identification. 
Table 2: Parameters of the bistable harvester prototype.

\begin{tabular}{lccc}
\hline \hline Parameter & Symbol & Value & Unit \\
\hline Stable positions & $\pm x_{0}$ & \pm 0.50 & $\mathrm{~mm}$ \\
Mass-frame distance & $L$ & 25 & $\mathrm{~mm}$ \\
Inertial mass & $M$ & 17.3 & $\mathrm{~g}$ \\
Natural angular frequency & $\omega_{0}$ & 121 & $\mathrm{rad}^{-1}$ \\
Mechanical quality factor & $Q$ & 87 & - \\
Excitation magnitude & $A$ & 2.5 & $\mathrm{~m}^{-2}$ \\
Excitation frequency & $\omega$ & $15-85$ & $\mathrm{~Hz}$ \\
Piezo converter force factor & $\alpha$ & 0.068 & $\mathrm{~N} . \mathrm{V}^{-1}$ \\
Piezo converter blocked capacity & $C_{0}$ & 1.05 & $\mu \mathrm{F}$ \\
Load resistance & $R$ & 7.83 & $\mathrm{k} \Omega$ \\
Electromechanical coupling factor & $k_{\mathrm{em}}^{2}$ & 2.74 & $\%$ \\
& & &
\end{tabular}

\section{III.2. Experimental results}

The frequency response obtained with the mathematical model is here compared with the frequency response obtained experimentally in order to control its relevance.

This experimental frequency response is determined as follows. The prototype is placed on a shaker ensuring a sinusoidal acceleration of constant amplitude $2.5 \mathrm{~m} . \mathrm{s}^{-2}$ The position of the mass and the position of the frame are measured using two laser vibrometers. The frequency ranges of harmonic 1 and subharmonic behaviors of the bistable harvester are determined with the following method: (i) The frequency of the excitation is set to a value where the behavior under study exists (based on theoretical predictions); (ii) Square voltage pulses are applied to the piezoelectric converter $(30 \mathrm{~V}$ for $20 \mathrm{~ms})$ thus generating force pulses on the mass. Each force pulse disturbs the current steady-state behavior and are likely to lead to another steady-state behavior (orbit jump), including possible subharmonics. These pulses are applied until the studied behavior is reached; (iii) Once the desired behavior is reached, the excitation frequency is slowly and smoothly increased or decreased in order to explore its entire frequency range without destabilizing it. The experimental setup is shown in Figure 8.

Figure 9 shows the theoretical and experimental fre-

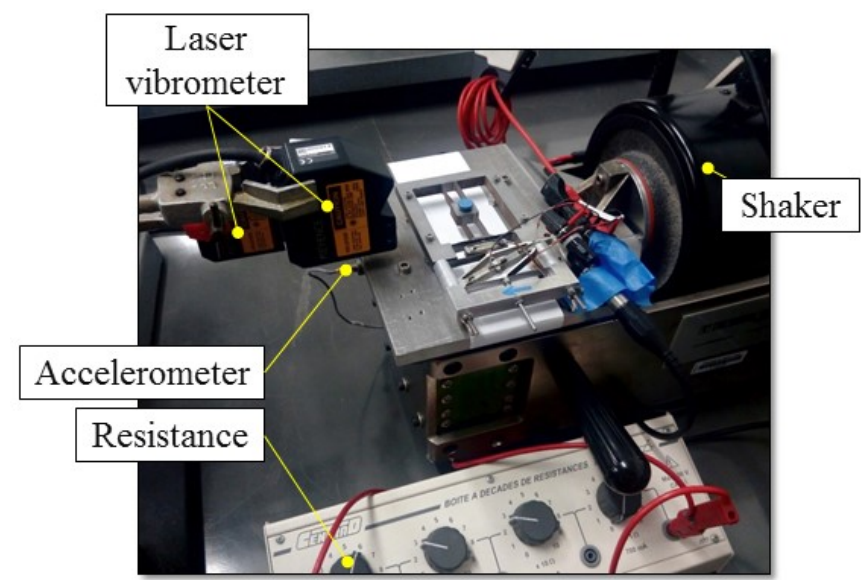

Figure 8: Setup used to determine the experimental frequency response of the prototype. The latter is mounted on a shaker ensuring a sinusoidal acceleration of constant amplitude $2.5 \mathrm{~m} \cdot \mathrm{s}^{-2}$. quency response of the piezoelectric bistable harvester. Only the theoretical behaviors defined as stable to small disturbances are drawn on this figure (the unstable behaviors are not interesting for energy harvesting as they cannot be reached experimentally). The stable behaviors are split in two different categories depending on the stability robustness criterion: robust enough or not robust enough to be maintained over time in real conditions. Each theoretical and experimental behavior are characterized by four items: the amplitude of the mass oscillation (greater than $x_{0}$ for high orbits and lower than $x_{0}$ for low orbits), the phase of the excitation displacement when the position of the mass reaches a maximum (for high orbits), the stability robustness (for high orbits) and finally the mean harvested power $\left(P_{\text {mean }}=v_{r m s}^{2} / R\right)$. The stability robustness threshold has been chosen in such a way that the cutting frequencies of the different high orbits match with the experimental observations.

The phase of the excitation displacement when the position of the mass reaches a maximum (equivalent to the phase shift for harmonic 1 behavior) is calculated considering that the mass displacement signal can be approached by its fundamental frequency. In other words, it is supposed that the instant on which the mass reaches a maximum does not change if we only take the fundamental frequency. This assumption is illustrated with few examples shown in Figure 10 (numerical study). For subharmonic $n$ high orbit, the fundamental component of the displacement is:

$$
\begin{aligned}
x_{\text {fundamental }}(t) & =a_{1} \cos \left(\frac{\omega}{n} t\right)+b_{1} \sin \left(\frac{\omega}{n} t\right) \\
& =\sqrt{a_{1}^{2}+b_{1}^{2}} \cos \left(\frac{\omega}{n} t+\psi\right)
\end{aligned}
$$

with

$$
\psi=\tan ^{-1}\left(\frac{-b_{1}}{a_{1}}\right)+\pi\left(\text { if } a_{1}<0\right)
$$

The instant on which the mass position reaches a maximum is called $t_{1}$. Hence, the phase of the mass position at $t_{1}$ is equal to zero:

$$
\begin{aligned}
\frac{\omega}{n} t_{1}+\psi & =0 \quad \bmod [2 \pi] \\
\Rightarrow t_{1} & =-\psi \frac{n}{\omega} \quad \bmod \left[2 \pi \frac{n}{\omega}\right]
\end{aligned}
$$

The excitation displacement can be written as $A / \omega^{2} \cos (\omega t+\pi)$. The phase of the excitation displacement when the position of the mass reaches a maximum (at $t_{1}$ ) thus becomes:

$$
\begin{array}{rlr}
\text { Phase } & =\omega t_{1}+\pi \quad \bmod [2 \pi] \\
& =-n \psi+\pi \quad \bmod [2 \pi]
\end{array}
$$

If this phase is zero, the displacement of the excitation reaches a maximum when the position of the mass reaches a maximum. If this phase is $\pi$, the displacement of the excitation reaches a minimum when the position of the mass reaches a maximum. As the calculated phase 

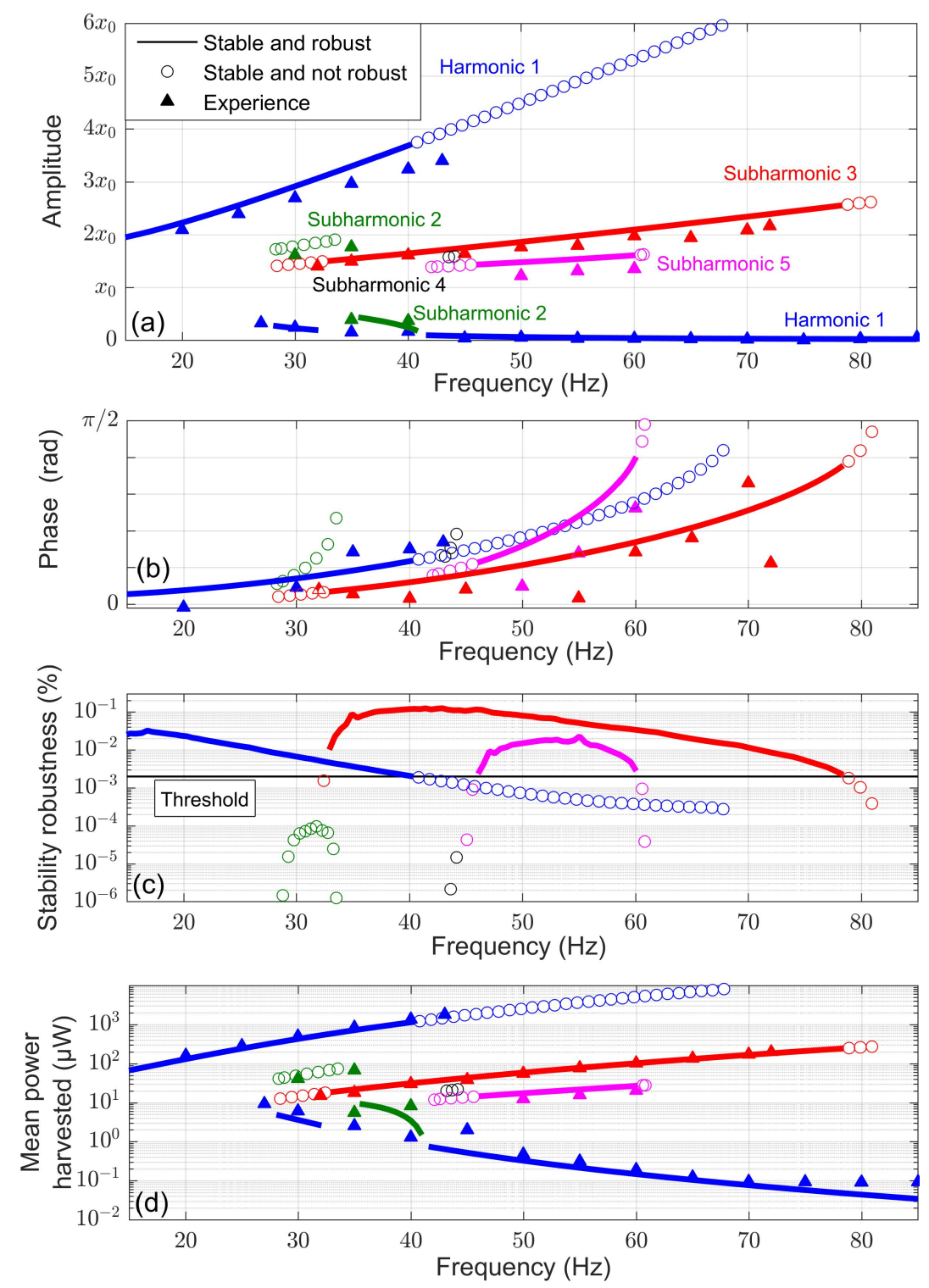

Figure 9: Theoretical and experimental spectra of the bistable harvester for a sinusoidal excitation of amplitude 2.5 m.s ${ }^{-2}$ : (a) amplitude of the different behaviors (b) phase of the excitation displacement when the position of the mass reaches a maximum (high orbits) (c) stability robustness (high orbits) (d) mean harvested power.

is the phase of the excitation displacement, the result is generally positive: the frame displacement is ahead of the mass displacement.

In the model, the Fourier series of the different behaviors ( $x$ and $v$ ) were truncated to order $N=6$ (to justify this choice, the reader can refer to the examples shown in Figure 5). For high orbits, subharmonics of order greater than five are not represented because they are not interesting for energy harvesting (their energy level and stability robustness are low compared to those of the behaviors plotted in Figure 9). Low orbits are not interesting for energy harvesting either. However, some of them are plotted in Figure 9 in order to know all the frequency ranges on which high orbits coexist with a low orbit. In these frequency ranges, high orbits may therefore fall on a low orbit, thus reducing the amount of energy converted by the bistable harvester.
Figure 9 shows a good agreement between the theoretical model and the experimental data. The frequency ranges of the different behaviors, their amplitude and their phases are well predicted by the theory when the stability robustness criterion is taken into account. The experimental data therefore confirm the relevance of this mathematical model which will then be exploited in the next section in order to give some guidelines for optimization of future bistable harvester prototypes.

\section{Model exploitation for optimized bistable harvesters}

The previous sections have introduced a theoretical model for piezoelectric bistable harvesters able to predict their frequency response including harmonic 1 and subharmonic behaviors. This model will now be exploited 

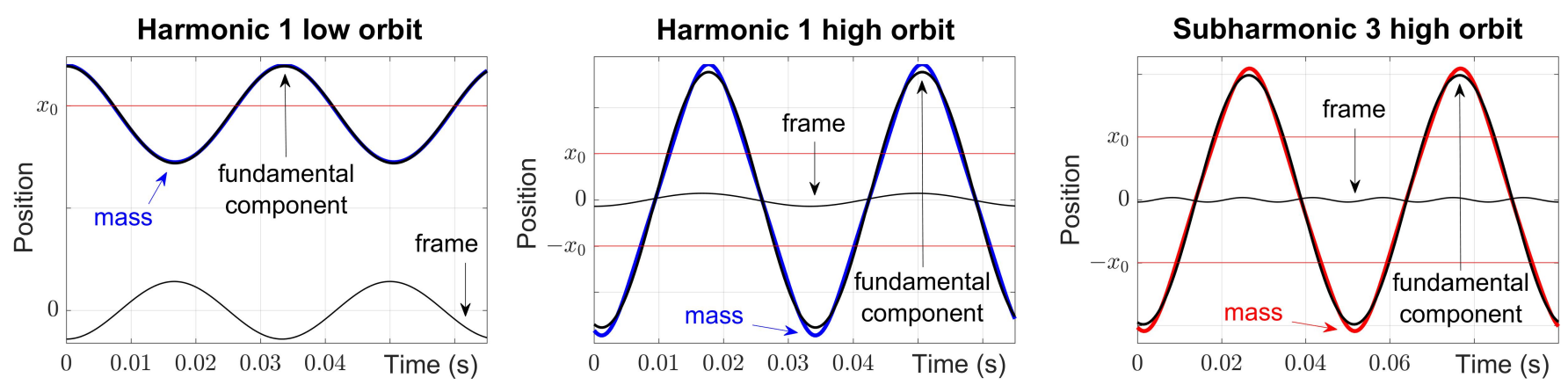

Figure 10: Mass position signal (polychromatic) compared to its fundamental frequency for harmonic 1 high and low orbit (obtained numerically for a 30 Hz excitation) and for subharmonic 3 high orbit (obtained numerically for a $60 \mathrm{~Hz}$ excitation).

to investigate the influence of different parameters (mass, stiffness, buckling level) on this frequency response to offer some leads to optimize future prototypes. A comparison with linear harvesters is also proposed all along this analysis.

The set of parameters chosen to fully describe the bistable harvester is $\left(M, k, x_{0}, L, \mu, R, \alpha, C_{0}\right)$. With this set, the general equations governing the behavior of the piezoelectric bistable harvester with low buckling level (Equation (5)) become:

$$
\left\{\begin{array}{l}
\ddot{x}+\frac{x_{0}^{2}}{L^{2}} \frac{2 k}{M}\left(\frac{x^{2}}{x_{0}^{2}}-1\right) x+\frac{\mu}{M} \dot{x}+\frac{2 \alpha}{M L} x v=-A \cos (\omega t) \\
C_{0} \dot{v}=2 \frac{\alpha}{L} x \dot{x}-\frac{v}{R}
\end{array}\right.
$$

In the following, not all these parameters will be considered as variable, but only the relevant ones, as explained in the followings. First, $x_{0}$ is bijectively coupled to the length $L$ : a value of $x_{0}$ is only obtained for a single value of $L$ and vice-versa. Therefore, the length $L$ will not be considered here as its influence can be deduced from the influence of $x_{0}$. The coefficient $\mu$ mostly reflects the friction of the air on the mass. This coefficient does not need to be studied: it has to be reduced as much as possible to reduce the mechanical losses in the harvester. It will therefore not be considered in the following study. Instead of $\alpha$ and $C_{0}$, the piezoelectric converter will be characterized by a more global parameter, namely the electromechanical coupling factor $k_{\mathrm{em}}^{2}$, representing its ability to convert the mechanical energy supplied by the source into electrical energy. It is defined in open circuit $(I=0)$ for a quasi-static regime (the kinetic energy of the mass is low in front of its elastic energy) by the following expression:

$$
k_{\mathrm{em}}^{2}=\frac{\text { Electrostatic energy stored in the piezo }}{\text { Mechanical energy supplied by the source }}
$$

The electromechanical coupling factor is therefore linked to two different aspects: (i) the ratio between the elastic energy stored in the piezoelectric material used in the harvester and the elastic energy stored in the other materials; (ii) the quality of this piezoelectric material (more or less able to convert its elastic energy into electrical energy). The electromechanical coupling factor is considered near-optimal for our prototype: the latter cannot be increased significantly. Indeed, (i) the piezoelectric material used is PZT (in 33 direction) which has the best ratio quality/price of nowadays market; (ii) the materials (other than piezoelectric) storing some unconverted elastic energy during the deformations are the flexible beams and the elliptical shell of the piezoelectric stacks. The first one stores a negligible quantity of elastic energy compared to the quantity stored in the piezoelectric element. The second one stores a bigger quantity of elastic energy but this quantity is cannot be decreased without affecting the mechanical functions that this shell must satisfy (hold the piezoelectric stacks, transfer the stresses, etc.). Hence, the electromechanical coupling factor of the prototype is supposed to be optimal for the studied architecture. For bistable harvesters, the analytical expression of this electromechanical coupling factor as a function of $\alpha$ and $C_{0}$ is given by Equation (26).

The remaining parameters are $\left(M, k, x_{0}, R\right)$ which can all be easily changed on the prototype: $M$ with the size of the mass, $k$ with the ratio between the small axis and the long axis of the piezoelectric shell, $x_{0}$ with the buckling level of the harvester and $R$ with the load plugged to the piezoelectric converter.

Table 3 summarizes the set of parameters chosen to fully describe the bistable harvester, highlights those considered as changeable in this study (stars) and details the values which correspond to the prototype introduced in the experimental section.

Figures 15, 11, 12 and 13 show the influence of these

\begin{tabular}{|c|c|c|c|c|}
\hline & Parameter & Symbol & Proto & Unit \\
\hline & Mass-frame distance & $L$ & 25 & $\mathrm{~mm}$ \\
\hline & Damping & $\mu$ & 0.023 & $\mathrm{~N} / \mathrm{m} / \mathrm{s}$ \\
\hline & Blocked capacity & $\mathrm{C}_{0}$ & 1.05 & $\mu \mathrm{F}$ \\
\hline & Electromechanical coupling factor & $k_{\mathrm{em}}^{2}$ & 2.74 & $\%$ \\
\hline * & Inertial mass & $M$ & 17.3 & $\mathrm{~g}$ \\
\hline * & Stiffness & $k$ & 159 & $\mathrm{kN} / \mathrm{m}$ \\
\hline * & Stable positions & $\pm x_{0}$ & \pm 0.50 & $\mathrm{~mm}$ \\
\hline * & Load resistance & $R$ & $7 s .83$ & $\mathrm{k} \Omega$ \\
\hline
\end{tabular}

Table 3: Parameters describing the bistable harvester.

* parameters considered as easily changeable. 
changeable parameters on the frequency response of the bistable harvester while the other parameters are kept constant. Only two behaviors are studied on these figures. These are the two most relevant behaviors for vibration energy harvesting: harmonic 1 high orbit (on which the working point maximizing the mean harvested power is located) and subharmonic 3 high orbit (offering a useful frequency range complementary to that of harmonic 1). Each of them is characterized by three quantities: the amplitude of oscillations of the mass, the stability robustness and the mean harvested power.

Despite its importance to define the behaviors of the bistable harvester, the phase of the excitation displacement when the position of the mass reaches a maximum has not been represented on these figures. The readability of the graph representing the latter is indeed not good (the curves are mixed and confused). For odd subharmonic behaviors, this phase always begins at 0 and grows monotonically until reaching $\pi / 2$ for the theoretical cutting frequency (see Figure 9). The following solution was chosen in order to make this criterion appear without reducing the readability of the figures: the working points for which this phase is $\pi / 2$ are represented by solid circles. These solid circles therefore correspond to the theoretical upper limit (cutting frequency) of the different behaviors.

Lastly, the frequency response of the equivalent linear harvester is also plotted in the following figures so that the performance of these two kinds of harvesters can be compared. This equivalent linear harvester has the same mass $M$, the same damping coefficient $\mu$, the same excitation $A \cos (\omega t)$, the same natural angular frequency $\omega_{0}$ and the same electromechanical coupling factor $k_{\mathrm{em}}^{2}$ as the bistable harvester. Thus, the equivalent linear harvester is governed by the following behavior law:

$$
\left\{\begin{array}{l}
\ddot{x}+\omega_{0}^{2} x+\frac{\mu}{M} \dot{x}+\frac{\alpha_{\operatorname{lin}}}{M} v=-A \cos (\omega t) \\
C_{0} \dot{v}=\alpha_{\operatorname{lin}} \dot{x}-\frac{v}{R}
\end{array}\right.
$$

with (as detailed in Equation (6))

$$
\omega_{0}=\frac{x_{0}}{L} \sqrt{\frac{4 k}{M}}
$$

Moreover, the electromechanical coupling factor of a linear harvester is given by the following expression [14]

$$
\begin{aligned}
k_{\mathrm{em}}^{2} & =\frac{\alpha_{\mathrm{lin}}^{2}}{M \omega_{0}^{2} C_{0}} \\
\Longrightarrow \quad \alpha_{\mathrm{lin}} & =\sqrt{k_{\mathrm{em}}^{2} M \omega_{0}^{2} C_{0}}
\end{aligned}
$$

\section{IV.1. Influence of $M$}

Figure 11 shows the influence of the mass $M$ on the frequency response of the bistable harvester. The value of the other parameters correspond to the prototype introduced in the experimental section. They are detailed in Table 3. For each mass, the load resistance is set to ensure the impedance matching in order to maximize the maximal mean harvested power: $R_{\text {match }}=1 /\left(2 C_{0} \omega_{0}\right)$ for the bistable harvester and $R_{\text {match }}=1 /\left(C_{0} \omega_{0}\right)$ for the linear harvester (for the bistable harvester the voltage of the piezoelectric converter oscillates at a frequency twice higher than the excitation as illustrated in Figure 3 and 5).

When the mass $M$ increases: (1) the amplitude of the mass oscillations for a given excitation frequency increases for both behaviors; (2) their theoretical cutting frequency (defined by a phase of $\pi / 2$ ) also increases resulting in an increase in the theoretical bandwidth of the harvester; (3) their real cutting frequency (defined by the stability robustness limit) decreases slightly for harmonic behavior 1 and increases slightly for subharmonic behavior 3 resulting in a slight overall increase in the global bandwidth of the harvester in real conditions; (4) this is explained by the stability robustness which increases for both behaviors with the mass; (5) the mean harvested power for a given excitation frequency increases; (6) the maximum harvested power (theoretical and real) also increases.

It is then preferable to increase the mass $M$ as much as possible (within the limits of the volume constraints imposed) in order to increase the mean harvested power of future bistable harvesters without significantly affecting its global frequency bandwidth.

\section{IV.2. Influence of $k$}

Figure 12 shows the influence of the stiffness $k$ on the frequency response of the bistable harvester. The value of the other parameters correspond to the prototype introduced in the experimental section. They are detailed in Table 3. For each stiffness, the load resistance is set to ensure the impedance matching in order to maximize the maximal mean harvested power: $R_{\text {match }}=1 /\left(2 C_{0} \omega_{0}\right)$ for the bistable harvester and $R_{\text {match }}=1 /\left(C_{0} \omega_{0}\right)$ for the linear harvester.

When the stiffness $k$ increases: (1) the amplitude of the mass oscillations for a given excitation frequency decreases for both behaviors; (2) their theoretical cutting frequency (defined by a phase of $\pi / 2$ ) increases; (3) their real cutting frequency (defined by the stability robustness limit) increases; (4) the stability robustness reaches a lower maximum value for both behaviors but reaches the robustness limit for a higher frequency; (5) The mean harvested power for a given excitation frequency decreases; (6) the maximum harvested power (theoretical and real) remains constant for harmonic 1 high orbit and slightly increases for subharmonic 3 high orbit.

Globally speaking, increasing the stiffness $k$ leads to a shift of the power curves towards higher frequencies. 
The stiffness can therefore be used (adjusting the ratio between the small axis and the long axis of the piezoelectric shell) to optimize the frequency range offered by the bistable harvester in such a way that it includes all the potential frequencies of the targeted vibration source. This adjustment must remain tight so that the robustness of the behaviors is not reduced too much.

\section{IV.3. Influence of $x_{0}$}

Figure 13 shows the influence of the stable position value $x_{0}$ on the frequency response of the bistable harvester. The value of the other parameters correspond to the prototype introduced in the experimental section. They are detailed in Table 3. For each value, the load resistance is set to ensure the impedance matching in order to maximize the maximal mean harvested power: $R_{\text {match }}=1 /\left(2 C_{0} \omega_{0}\right)$ for the bistable harvester and $R_{\text {match }}=1 /\left(C_{0} \omega_{0}\right)$ for the linear harvester.

When the value of the stable positions $x_{0}$ increases: (1) the amplitude of the mass oscillations for a given excitation frequency increases for both behaviors; (2) the theoretical cutting frequency (defined by a phase of $\pi / 2$ ) of harmonic 1 high orbit decreases while it remains relatively constant for subharmonic 3 high orbit; (3) their real cutting frequency (defined by the stability robustness limit) behave in the same way. Globally, the real frequency range of both behaviors decreases when $x_{0}$ increases. The frequency range of harmonic 1 high orbit is however more affected than that of subharmonic 3 high orbit. (4) The stability robustness decreases for both behaviors; (5) the mean harvested power for a given excitation frequency increases; (6) the maximum harvested power (theoretical and real) remains constant for har-
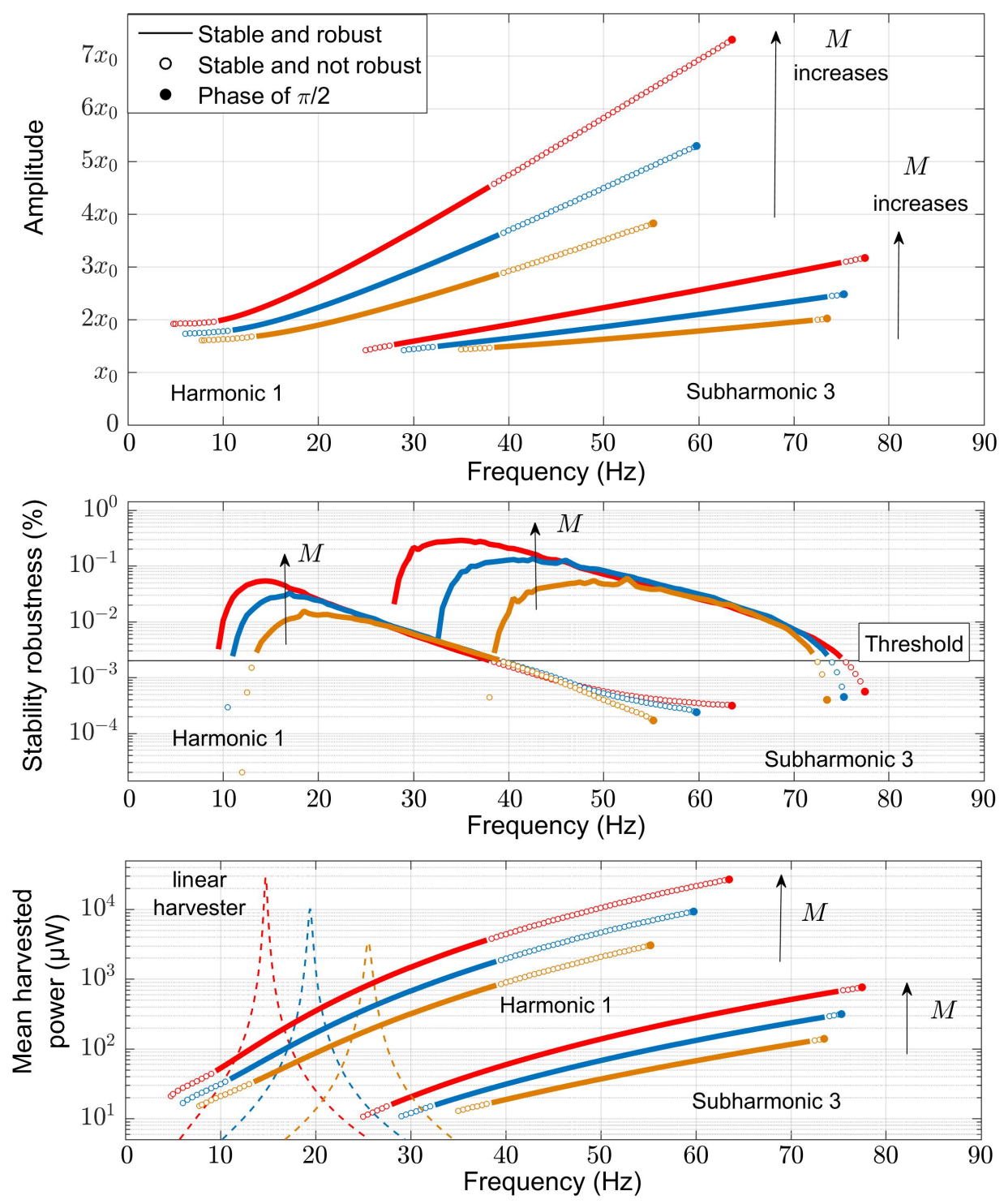

Figure 11: Influence of the mass $M$ on the frequency response of the bistable harvester for an excitation of $2.5 \mathrm{m.s} \mathrm{s}^{-2} . \mathrm{Masses}$ shown: $10 \mathrm{~g}$ (orange curves), $17.3 \mathrm{~g}$ (prototype value - blue curves) and $30 \mathrm{~g}$ (red curves). These values receptively correspond to the following natural angular frequencies: 159 rad.s ${ }^{-1}$ $(25 \mathrm{~Hz}), 121 \mathrm{rad} . \mathrm{s}^{-1}(19 \mathrm{~Hz})$ and $92 \mathrm{rad} . \mathrm{s}^{-1}(15 \mathrm{~Hz})$. For each mass, the load resistance is set to ensure the impedance matching $R_{\text {match }}=1 /\left(2 \mathrm{C}_{0} \omega_{0}\right): 2.98 \mathrm{k} \Omega$, $3.91 \mathrm{k} \Omega$ and $5.16 \mathrm{k} \Omega$. For each behavior, the theoretical cutting frequency is reached when the phase of the phase of the excitation displacement is $\pi / 2$ when the position of the mass reaches a maximum. The response of the equivalent linear harvester is shown in dashed curves. 
monic 1 high orbit and increases for subharmonic 3 high orbit.

From these observations, it can be interesting to increase the value of the stable positions $x_{0}$ (changing the buckling level of the harvester) until the overlap area between harmonic 1 and subharmonic 3 behaviors disappears. The mean harvested power would therefore be globally increased without losing too much bandwidth. For our prototype, the best configuration with this approach corresponds to the blue curves in Figure 13.

\section{IV.4. Influence of $R$}

The optimization of the parameter $R$ is more specific than the other parameters studied above as it does not concern the mechanical harvester itself but the electrical circuit connected to its piezoelectric converter. Figure 14 shows the influence of $R$ on harmonic 1 high orbit and subharmonic 3 high orbit. The value of the other parameters correspond to the prototype introduced in the experimental section. They are detailed in Table 3. The maximum mean harvested power of both behaviors considering stable orbits (solid lines + circles) is optimal when the load resistance is set to match the impedance of the piezoelectric converter at the natural angular frequency $\omega_{0}$ of the harvester (red curves). This resistance is equal to $R_{\text {match }}=1 /\left(2 C_{0} \omega_{0}\right)$ for bistable harvesters as the voltage of the piezoelectric converter oscillates at a frequency twice higher than the excitation (phenomenon illustrated in Figure 3 and 5). When the load resistance is increased or decreased, the maximum mean harvested power of both behaviors decreases while their respective frequency ranges increases.
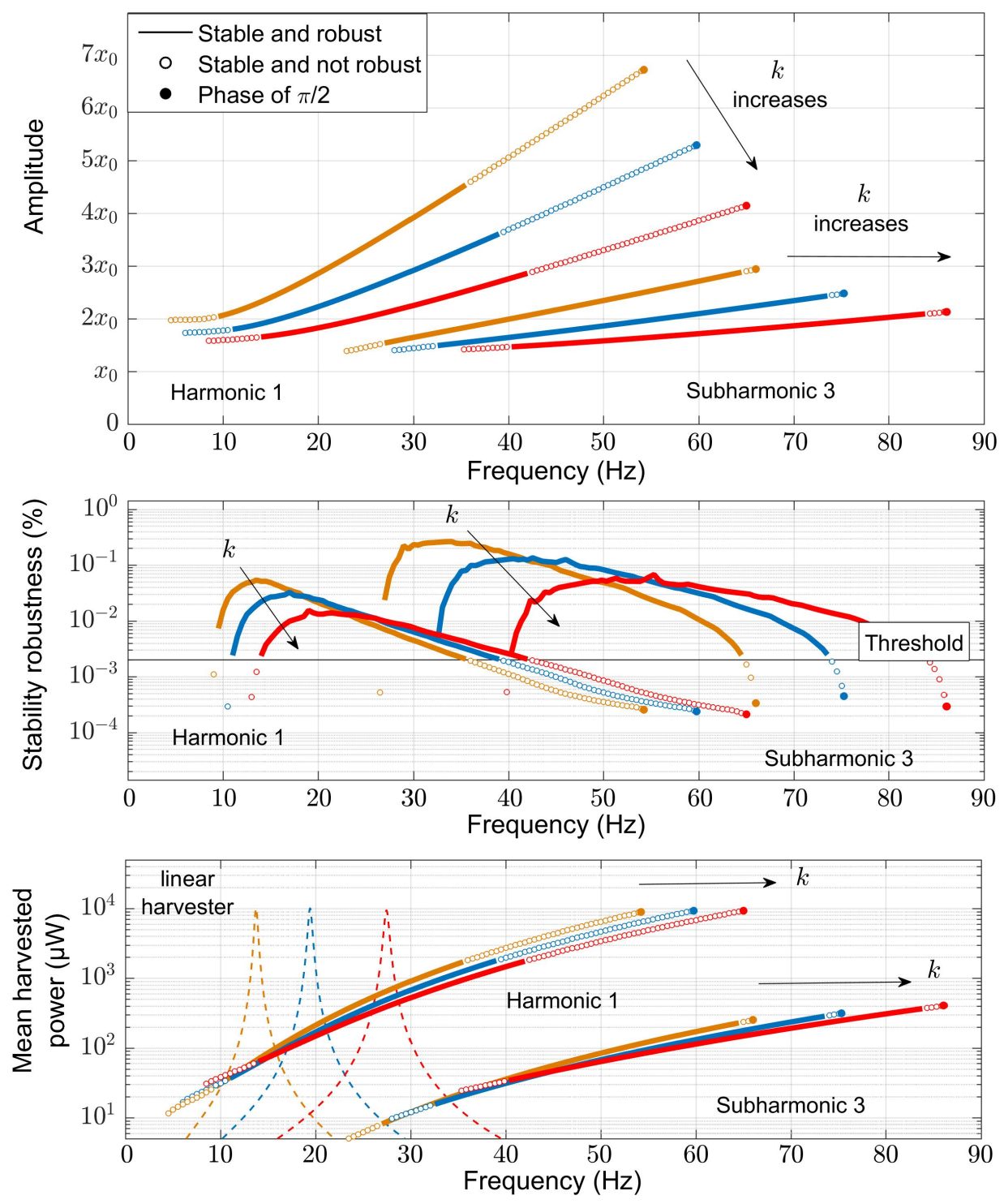

Figure 12: Influence of the stiffness $k$ on the frequency response of the bistable harvester for an excitation of $2.5 \mathrm{~m} . \mathrm{s}^{-2}$. Stiffness shown: $79.5 \mathrm{kN} . \mathrm{m}^{-1}$ (orange curves), $159 \mathrm{kN} \cdot \mathrm{m}^{-1}$ (prototype value - blue curves) and $318 \mathrm{kN} . \mathrm{m}^{-1}$ (red curves). These values receptively correspond to the following natural angular frequencies: $86 \mathrm{rad} . \mathrm{s}^{-1}(14 \mathrm{~Hz}), 121 \mathrm{rad} . \mathrm{s}^{-1}(19 \mathrm{~Hz}), 171 \mathrm{rad} . \mathrm{s}^{-1}(27 \mathrm{~Hz})$. For each mass, the load resistance is set to ensure the impedance matching $R_{\text {match }}=1 /\left(2 C_{0} \omega_{0}\right): 5.54 \mathrm{k} \Omega, 3.91 \mathrm{k} \Omega$ and $2.77 \mathrm{k} \Omega$. For each behavior, the theoretical cutting frequency is reached when the phase of the phase of the excitation displacement is $\pi / 2$ when the position of the mass reaches a maximum. The response of the equivalent linear harvester is shown in dashed curves. 
Figure 15 shows a comparison between the frequency responses of the bistable harvester given by the orbits defined as stable and robust obtained with $R_{\text {match }}$ (red curves) and obtained with the resistance tuned (grey curves) to ensure the highest mean harvested power for each frequency. The resistance tuning is profitable on both harmonic 1 high orbit (maximal mean harvested power increased by $20 \%$ and frequency range increased by $14 \%$ ) and on subharmonic 3 high orbit (frequency range increased by $49 \%$ ). The global frequency range offered by the bistable harvester is increased by $32 \%$ with the resistance tuning.

In conclusion, the optimal resistance to maximize the mean harvested power over the entire frequency range of the bistable harvester is obtained when it is set equal to the impedance of the piezoelectric converter at the natural angular frequency $\omega_{0}$ of the bistable harvester:
$R_{\text {match }}=1 /\left(2 C_{0} \omega_{0}\right)$ (red curves in Figure 14). Then, a resistance tuning when the excitation get beyond the real cutting frequencies (defined by the stability robustness limit) of the two behaviors will allow to increases the global frequency range offered by the harvester by roughly $30 \%$ and increase its maximal mean harvested power by roughly $20 \%$.

\section{IV.5. Bistable vs linear}

The first observation that can be made from the comparison between linear and bistable harvesters in Figures 11, 12 and 13 concerns the mean harvested power: the maximum reached with the linear harvester (at its resonance frequency) is systematically equal to the maximum reached with the bistable generator (at the theoretical cutting frequency of the harmonic 1 high orbit
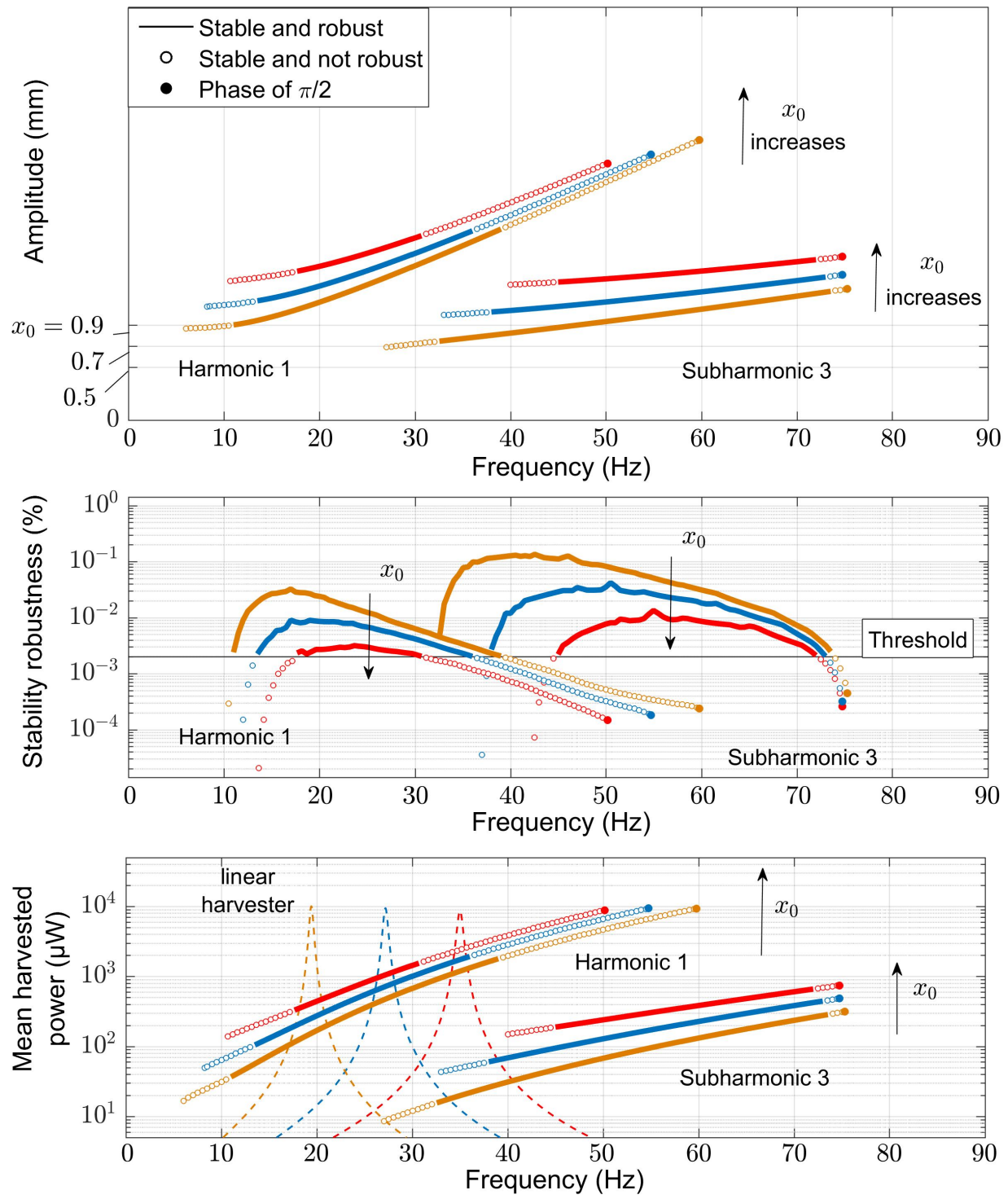

Figure 13: Influence of the stable positions value $x_{0}$ on the frequency response of the bistable harvester for an excitation of $2.5 \mathrm{~m} . \mathrm{s}^{-2}$. Values shown: $0.50 \mathrm{~mm}$ (prototype value - orange curves), $0.70 \mathrm{~mm}$ (blue curves) and $0.90 \mathrm{~mm}$ (red curves). These values receptively correspond to the following natural angular frequencies: 121 rad.s $\mathrm{s}^{-1}(19 \mathrm{~Hz}), 170 \mathrm{rad} . \mathrm{s}^{-1}(27 \mathrm{~Hz}), 218 \mathrm{rad} . \mathrm{s}^{-1}(35 \mathrm{~Hz})$. For each mass, the load resistance is set to ensure the impedance matching $R_{\text {match }}=1 /\left(2 C_{0} \omega_{0}\right): 3.91 \mathrm{k} \Omega, 2.80 \mathrm{k} \Omega$ and $2.18 \mathrm{k} \Omega$. For each behavior, the theoretical cutting frequency is reached when the phase of the phase of the excitation displacement is $\pi / 2$ when the position of the mass reaches a maximum. The response of the equivalent linear harvester is shown in dashed curves. 


\section{Harmonic 1 high orbit}

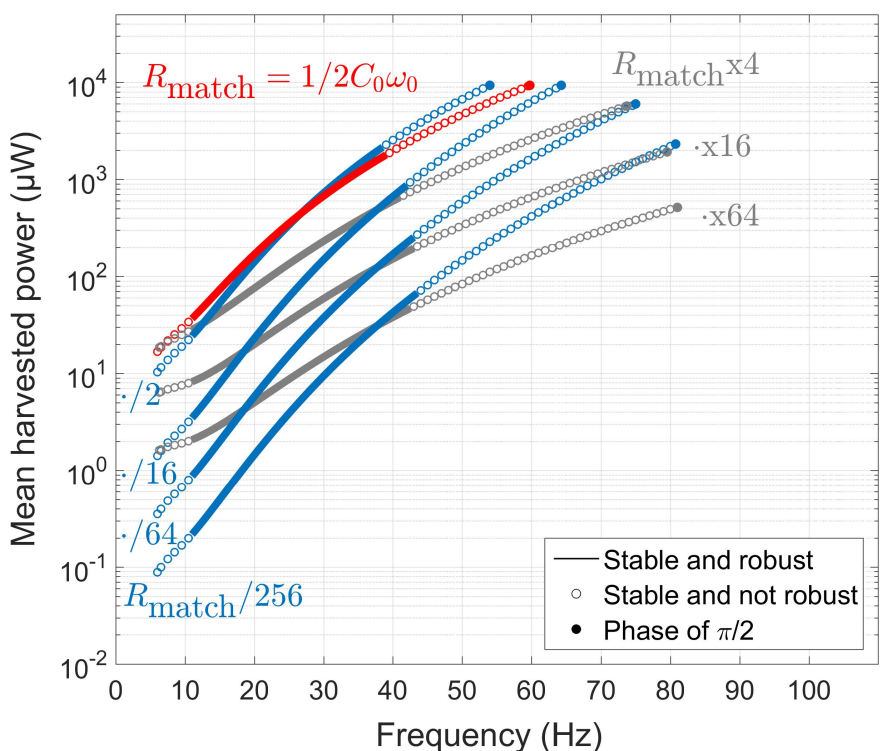

Subharmonic 3 high orbit

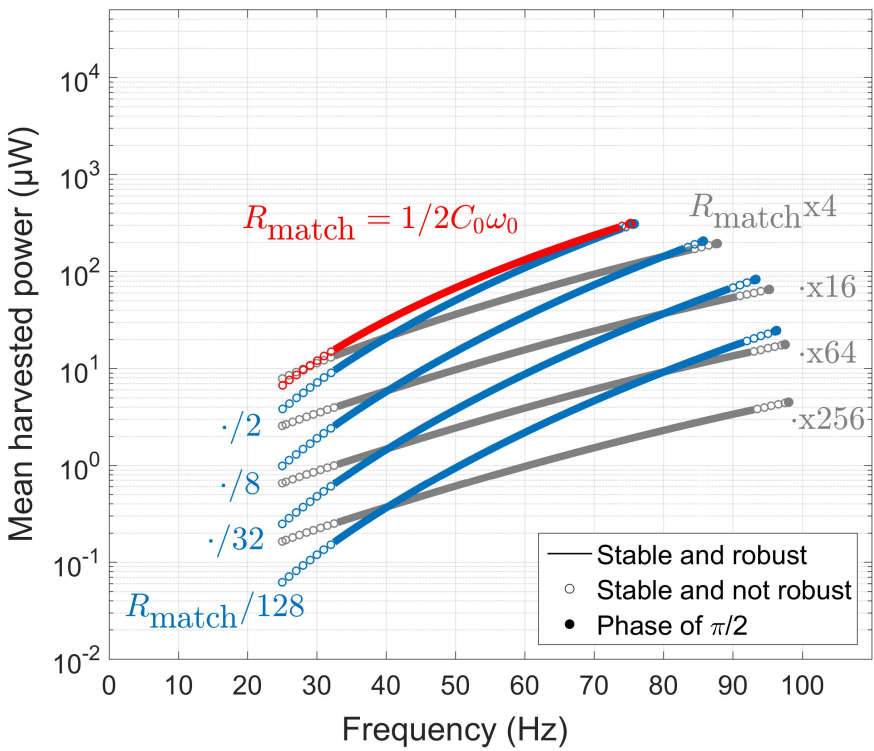

Figure 14: Influence of the load resistance $R$ on the behaviors of the bistable harvester for an excitation of $2.5 \mathrm{~m} . \mathrm{s}^{-2}$. Red curves correspond to the resistance ensuring the impedance matching with the piezoelectric converter for an excitation set at the natural angular frequency $R_{\text {match }}=1 /\left(2 C_{0} \omega_{0}\right)(3913 \Omega)$. Blue curves correspond to resistances lower than $R_{\text {match }}$ and grey curves higher than $R_{\text {match }}$. For each behavior, the theoretical cutting frequency is reached when the phase of the excitation displacement is $\pi / 2$ when the position of the mass reaches a maximum.
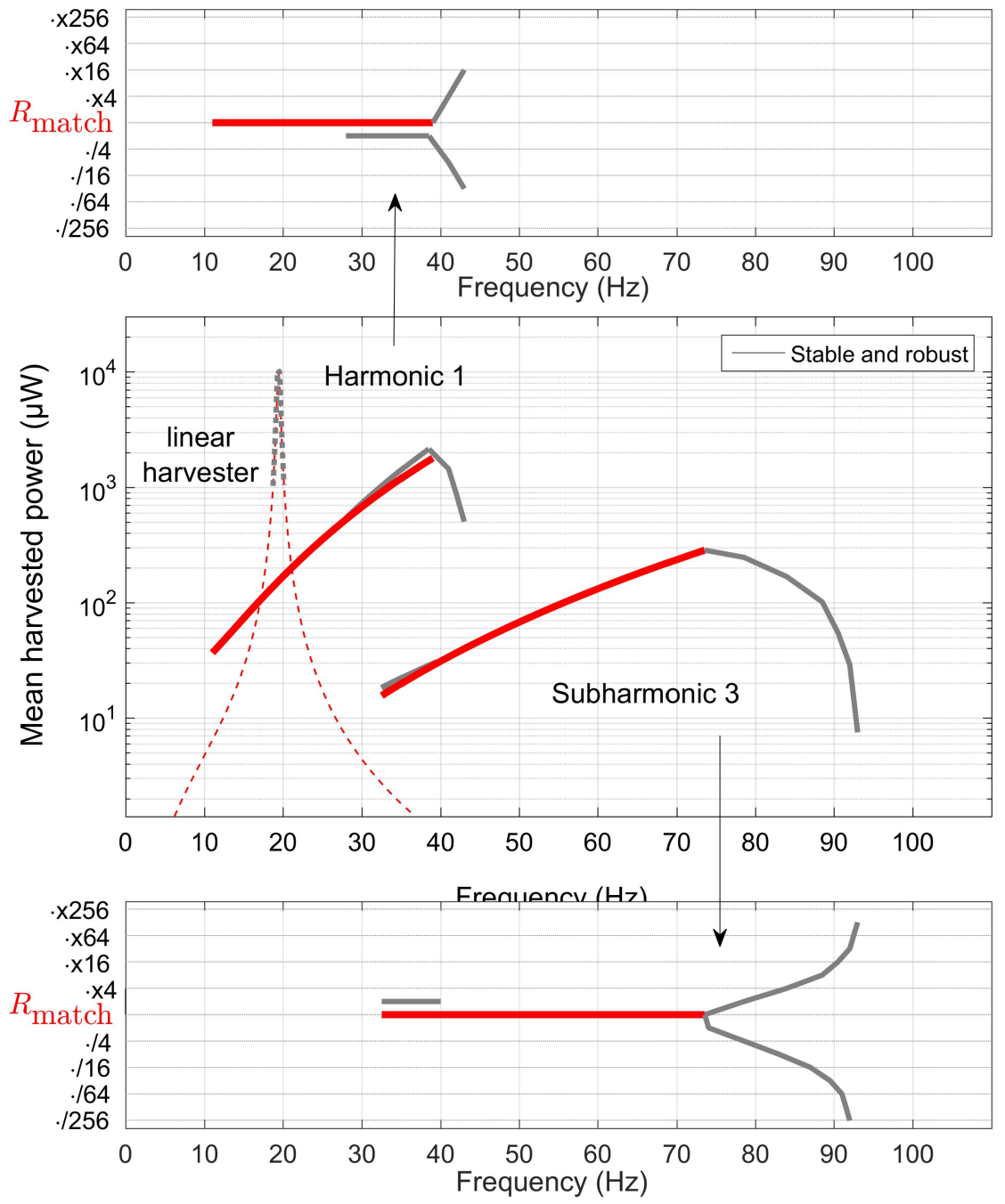

Figure 15: Frequency responses of the bistable harvester (excitation of $2.5 \mathrm{~m} . \mathrm{s}^{-2}$ ) obtained for (i) a resistance ensuring the impedance matching $R_{\text {match }}=$ $1 /\left(2 C_{0} \omega_{0}\right)$ (red curves) and (ii) for a resistance tuned to get the highest mean harvested power for each frequency (grey curves). The latter is plotted only when it leads to better performances than the impedance matching. The frequency responses of the linear harvester is plotted as reference (tuned in dashed grey and for the impedance matching $R=1 /\left(C_{0} \omega_{0}\right)$ in dashed red). 
defined by a phase of $\pi / 2$ ). Hence, in theory, those two harvesters have the same maximal performances.

However, the theoretical cutting frequency of the bistable harvester is not robust enough to be maintained over time in real conditions. The reachable maximum mean harvested power of the bistable harvester (robust orbits) is always lower than the maximum offered by the linear harvester. In terms of the maximum mean harvested power, the bistable harvester is therefore less efficient than its equivalent linear harvester [15].

On the other hand, the two harvesters do not have the same bandwidth. The frequency range on which the bistable harvester recovers more than $100 \mu \mathrm{W}^{1}$ on average is systematically bigger than linear harvester's one.

The stability robustness criterion therefore balances the interest of the bistable harvester for vibration energy harvesting. Its maximum mean harvested power is greatly reduced with this criterion and becomes much lower than that of the equivalent linear harvester. However, this weakness is counterbalanced by its large bandwidth compared to that of the linear harvester. The latter remains relevant even after the inclusion of the stability robustness criterion, partly due to the exploitation of subharmonic 3 behavior. The use of one or the other of these harvesters will therefore be linked to fluctuations in the frequency of the ambient vibration source. For a source with a fixed and stable frequency, the linear harvester will be more interesting. For a source with a fluctuating frequency, the bistable harvester will be preferred. This conclusion is different from that obtained by considering the classical theoretical study of bistable harvester (without stability robustness criteria) which would have favoured the bistable harvester in all cases (source with stable or fluctuating frequency).

Figures 11, 12, 13 and 15 also show how this comparison evolves with $\left(M, k, x_{0}, R\right)$. This evolution is summarized in Table 4 which introduces two different ratio for comparing the two harvesters: the ratio between their maximal mean harvested power and the ratio of their frequency range on which they harvest more than $100 \mu W^{1}$. For the bistable harvester, these data are measured on robust and stable orbits. When $M$ increases, the respective advantages of the two harvesters increase as well: the maximal mean harvested power of linear harvester increases relatively to bistable's one; the frequency range of bistable harvester increases relatively to linear's one. Decreasing $x_{0}$ is profitable for bistable harvester as its frequency range increase relatively to linear's ones while its maximal mean harvested power remains relatively constant. Increasing $k$ or tuning the resistance $R$ is profitable for bistable harvester as its maximal mean harvested power and its frequency range increase relatively

\footnotetext{
${ }^{1}$ With this average, it is possible to power an isolated wireless sensor consuming $450 \mathrm{~mJ}$ per transmitted packet $-22 \mathrm{~mA}$ at $3.3 \mathrm{~V}$ for $6.2 \mathrm{~ms}$ [16] - with one packet sent every $1.25 \mathrm{~h}$.
}

to linear's ones.

Table 4: Bistable vs linear.

\begin{tabular}{cccc}
\hline \hline Parameter & Value & Power ratio $^{*}$ & Freq. ratio $^{\circ}$ \\
\hline Mass & 30 & 0.13 & 10.8 \\
$M(\mathrm{~g})$ & 17.3 & 0.18 & 8.0 \\
& 10 & 0.24 & 5.8 \\
\hline Stiffness & 318 & 0.19 & 8.5 \\
$k(\mathrm{kN} / \mathrm{m})$ & 159 & 0.18 & 8.0 \\
& 79.5 & 0.17 & 7.8 \\
\hline Stable position & 0.90 & 0.18 & 5.7 \\
$x_{0}($ mm) & 0.70 & 0.20 & 8.3 \\
& 0.50 & 0.18 & 8.0 \\
\hline Resistance & $R_{\text {match }}$ & 0.18 & 8.0 \\
$R(\Omega)$ & tuned & 0.21 & 12.8 \\
\hline \hline
\end{tabular}

* ratio of maximal mean harvested powers (robust bistable/linear).

ratio of frequency ranges $>100 \mu W^{1}$ (robust bistable/linear).

\section{Conclusion}

The first part of this study introduced an analytical model for piezoelectric bistable harvester able to predict all its possible steady-state behaviors (including subharmonic behaviors) as a function of the excitation frequency. This model characterizes the different behaviors with two criteria: their stability to small disturbances and their stability robustness. The second criterion is calculated for stable high orbits and defines their ablity to handle bigger disturbances (that can be found in real conditions) without falling on a low orbit. This model has then be validated thanks to experimental data.

The second part of this study has exploited this model to investigate the influence of different parameters of the bistable harvester $\left(M, k, x_{0}\right)$ which can all be easily changed on future prototypes: $M$ with the size of the mass, $k$ with the ratio between the small axis and the long axis of the piezoelectric shell, $x_{0}$ with the buckling level of the harvester. The influence of the load resistance $R$ plugged to the piezoelectric converter has also been analyzed. Table 5 presents a summary of the results that have been obtained which can be used as a guide to design the future bistable harvesters with optimized performances for different potential applications. Considering the assumptions made in this article to get these results, these guidelines are valid for all the bistable harvesters which respect the two following constraints: (i) the behavioral equation must be a Duffing-type equation as detailed in Equation (5), (ii) the global electromechanical coupling must be relatively low. These two constraints are nevertheless satisfied by the majority of the bistable harveter prototypes presented so far in the literature [4].

Finally, this study focused on the comparison between bistable and linear harvesters. The stability robustness 
Table 5: Guidelines for optimized bistable harvester.

\begin{tabular}{|c|c|}
\hline Parameter & Action \\
\hline Inertial mass $M$ & $\begin{array}{l}\text { Increase as much as possible within the lim- } \\
\text { its of volume constraints imposed. }\end{array}$ \\
\hline Stiffness $k$ & $\begin{array}{l}\text { Adjust it to fit the frequency range on the } \\
\text { potential frequencies of the targeted vibra- } \\
\text { tion source (in the design introduced in the } \\
\text { article, adjust the ratio between the small } \\
\text { axis and the long axis of the piezoelectric } \\
\text { shell). This adjustment must remain tight so } \\
\text { that the robustness of the behaviors is not } \\
\text { reduced too much. }\end{array}$ \\
\hline Stable position $x_{0}$ & $\begin{array}{l}\text { Increase until the overlap area between har- } \\
\text { monic } 1 \text { and subharmonic } 3 \text { behaviors disap- } \\
\text { pears to increased the mean harvested power } \\
\text { without losing too much bandwidth. }\end{array}$ \\
\hline Load resistance $R$ & $\begin{array}{l}\text { Ensure the impedance matching with the } \\
\text { piezoelectric converter }\left(1 /\left(2 C_{0} \omega_{0}\right)\right) \text { to max- } \\
\text { imise the harvested power. } \\
\text { Tune it if the vibration source get beyond the } \\
\text { real cutting frequencies (defined by robust or- } \\
\text { bits) for both harmonic } 1 \text { and subharmonic } 3 \\
\text { behaviors to increase the global frequency } \\
\text { range by roughly } 30 \% \text { and the maximal mean } \\
\text { harvested power by roughly } 20 \% \text {. }\end{array}$ \\
\hline
\end{tabular}

criterion has balanced the interest of the bistable harvester for vibration energy harvesting. The maximum mean harvested power reachable by the bistable harvester is not as high as the common theory predicts and becomes much lower than the one reachable with the equivalent linear harvester. On the other hand, the bandwidth offered by the bistable harvester is much larger even after the inclusion of the stability robustness criterion (especially thanks to the use of subharmonic 3 behavior). One or the other of these harvesters will therefore be preferred depending on the vibration source. For a source presenting a high frequency fluctuation, the bistable harvester will show better results. For a source presenting a fixed and stable frequency, the linear harvester will be more relevant.

\section{Appendix}

The equations governing the behavior of bitable harvester detailed in Equation (5) is composed by a second order differential equation of the variable $x$ and a first order differential equation of the variable $v$. Hence, the initial conditions needed to solve the problem are $(x(0), \dot{x}(0), v(0))$. Table 6 details the initial conditions that lead to the behaviors presented in Figures 3, 4 and 5 thanks to a numerical study.
Table 6: Initial conditions for the numerical study.

\begin{tabular}{lcccc}
\hline \hline Behavior & $\begin{array}{c}\text { Freq. } \\
(\mathrm{Hz})\end{array}$ & $\begin{array}{c}x(0) \\
(\mathrm{mm})\end{array}$ & $\begin{array}{c}\dot{x}(0) \\
(\mathrm{mm} / \mathrm{s})\end{array}$ & $\begin{array}{c}v(0) \\
(\mathrm{V})\end{array}$ \\
\hline Harmonic 1 low orbit & 30 & -0.370 & 0 & -0.192 \\
Harmonic 1 high orbit & 30 & -1.37 & 0 & 2.02 \\
Subharmonic 2 high orbit & 30 & 0.911 & 0 & 1.06 \\
Subharmonic 3 high orbit & 50 & -0.909 & 0 & 1.03 \\
Subharmonic 4 high orbit & 44 & 0.634 & -44.1 & -0.128 \\
Subharmonic 5 high orbit & 50 & -0.730 & 0 & 0.500 \\
\hline \hline
\end{tabular}

\section{Acknowledgment}

The authors acknowledge the support of Région Auvergne-Rhône-Alpes through the ARC 4 Energies program.

\section{References}

[1] K.A. Cook-Chennault, N. Thambi, and A.M. Sastry. Powering MEMS portable devices: a review of nonregenerative and regenerative power supply systems with special emphasis on piezoelectric energy harvesting systems. Smart Materials and Structures, 17:043001, 2008.

[2] J. Siang, M.H. Lim, and M. Salman Leong. Review of vibration-based energy harvesting technology : Mechanism and architectural approach. International Journal of Energy Research, pages 1-28, 2018.

[3] M.F. Daqaq, R. Masana, A. Erturk, and D.D. Quinn. On the role of nonlinearities in vibratory energy harvesting: A critical review and discussion. Applied Mechanics Reviews, 66:40801, 2014.

[4] R.L. Harne and K.W. Wang. A review of the recent research on vibration energy harvesting via bistable systems. Smart Materials and Structures, 22:23001, 2013.

[5] G. Wang, W.H. Liao, B. Yang, X. Wang, W. Xu, and X. Li. Dynamic and energetic characteristics of a bistable piezoelectric vibration energy harvester with an elastic magnifier. Mechanical Systems and Signal Processing, 105:427 - 446, 2018.

[6] D. Liu, Y. Wu, X. Yong, and L. Jing. Stochastic response of bistable vibration energy harvesting system subject to filtered gaussian white noise. Mechanical Systems and Signal Processing, 130:201 - 212, 2019.

[7] C.B. Williams and R.B. Yates. Analysis Of A Microelectric Generator For Microsystems. Sensors and Actuators A: Physical, 52:8-11, 1996.

[8] T. Huguet, A. Badel, and M. Lallart. Exploiting bistable oscillator subharmonics for magnified broadband vibration energy harvesting. Applied Physics Letters, 111:173905, 2017. 
[9] T. Huguet, A. Badel, O. Druet, and M. Lallart. Drastic bandwidth enhancement of bistable energy harvesters: Study of subharmonic behaviors and their stability robustness. Applied Energy, 226:607-617, 2018.

[10] A.J. Sneller, P. Cette, and B.P. Mann. Experimental investigation of a post-buckled piezoelectric beam with an attached central mass used to harvest energy. Proceedings of the Institution of Mechanical Engineers, Part I: Journal of Systems and Control Engineering, 225:497-509, 2011.

[11] S.C. Stanton, B.A.M. Owens, and B.P. Mann. Harmonic balance analysis of the bistable piezoelectric inertial generator. Journal of Sound and Vibration, 331:3617-3627, 2012.

[12] M. Panyam, R. Masana, and M.F. Daqaq. On approximating the effective bandwidth of bi-stable energy harvesters. International Journal of Non-Linear Mechanics, 67:153-163, 2014.

[13] W.Q. Liu, A. Badel, F. Formosa, Y.P. Wu, and A. Agbossou. Novel piezoelectric bistable oscillator architecture for wideband vibration energy harvesting. Smart Materials and Structures, 22:035013, 2013.

[14] A. Badel and E. Lefeuvre. Nonlinear Conditioning Circuits for Piezoelectric Energy Harvesters. In E. Blokhina, A. El Aroudi, E. Alarcon, and D. Galayko, editors, Nonlinearity in Energy Harvesting Systems Nonlinear, pages 321-359. Springer, Cham, 2016.

[15] W.Q. Liu, A. Badel, F. Formosa, and Y.P. Wu. A new figure of merit for wideband vibration energy harvesters. Smart Materials and Structures, 24:125012, 2015.

[16] A. Gutiérrez, C. Gonzàlez, J. Jiménez-Leube, S. Zazo, N. Dopico, and I. Raos. A Heterogeneous Wireless Identification Network for the Localization of Animals Based on Stochastic Movements. Sensors, 9:3942-3957, 2009. 